\title{
FUNCTIONAL CALCULUS FOR DIAGONALIZABLE MATRICES
}

\author{
PIOTR NIEMIEC
}

\begin{abstract}
For an arbitrary function $f: \Omega \rightarrow \mathbb{C}$ (where $\Omega \subset \mathbb{C}$ ) and a positive integer $k$ let $f_{\text {op }}: \mathscr{D}_{k}(\Omega) \ni X \mapsto f[X] \in \mathscr{D}_{k}(\mathbb{C})$ where $\mathscr{D}_{k}(\Omega)$ consists of all $k \times k$ matrices similar to diagonal whose all eigenvalues lie in $\Omega$ be the function defined as follows: $f\left[P \operatorname{Diag}\left(\lambda_{1}, \ldots, \lambda_{k}\right) P^{-1}\right]=P \operatorname{Diag}\left(f\left(\lambda_{1}\right), \ldots, f\left(\lambda_{k}\right)\right) P^{-1}$ for arbitrary $\lambda_{1}, \ldots, \lambda_{k} \in \Omega$ and an invertible $k \times k$ matrix $P$. The aim of the paper is to fully answer the question of when $f_{\text {op }}$ is continuous for fixed $k$. In particular, it is shown that if $\Omega$ is open in $\mathbb{C}$, then $f_{\text {op }}$ is continuous for fixed $k \geqslant 3$ iff $f$ is holomorphic; and if $\Omega$ is an interval in $\mathbb{R}$ and $k \geqslant 3$, then $f_{\text {op }}$ is continuous on $\mathscr{D}_{k}(\Omega)$ iff $f \in C^{k-2}(\Omega)$ and $f^{(k-2)}$ is locally Lipschitz in $\Omega$. Also a full characterization is given when the domain of $f$ is arbitrary as well as when $f_{\text {op }}$ acts on infinite-dimensional (diagonalizable) matrices.
\end{abstract}

\section{INTRODUCTION}

Continuous (or Borel) functional calculus for normal operators is an interesting concept widely investigated in operator theory. There are many spectacular results dealing with this concept, e.g. Loewner's theorem [9] on operator monotone functions (for other proof and a discussion see Chapter V in [2]); Aleksandrov'sPeller's-Potapov's-Sukochev's theorem [1] on operator Hölder functions which turn out to coincide with Hölder functions - this is in contrast to Lipschitz functions which may not be operator Lipschitz (consult e.g. [8]). It is quite natural to extend the above functional calculus to other operators (or matrices) than normal. The simplest class applicable here is formed by diagonalizable matrices (that is, matrices similar to diagonal). In this way for any function $f: \Omega \rightarrow \mathbb{C}$ and each positive integer $k$ we may properly define a matrix-valued function $f_{\text {op }}: \mathscr{D}_{k}(\Omega) \rightarrow \mathscr{D}_{k}(\mathbb{C})$ where $f_{\text {op }}, \mathscr{D}_{k}(\Omega)$ and $\mathscr{D}_{k}(\mathbb{C})$ are as in Abstract. Having such an extended functional calculus, we may pose analogous questions as in case of normal matrices. Most basic among them is the continuity of $f_{\text {op }}$. More precisely, we may study the following issue:

1.1. Problem. Given a set $\Omega \subset \mathbb{C}$ and a positive integer $k$, characterize all functions $f: \Omega \rightarrow \mathbb{C}$ for which the function $f_{\text {op }}: \mathscr{D}_{k}(\Omega) \rightarrow \mathscr{D}_{k}(\mathbb{C})$ is continuous.

At first sight, one may suspect that the characterization is 'trivial', that is, that the continuity of $f$ is sufficient for the continuity of $f_{\text {op }}$. Surprisingly, it turns out that this supposition fails even for $k=2$. The main aim of the paper is to give a full answer to Problem 1.1. Our general characterization involves so-called divided differences (see Section 3). However, in most of practical cases, i.e. when the set $\Omega$ is open in $\mathbb{C}$ or a subinterval of $\mathbb{R}$, the criterion may simply be formulated. To this end, denote by $\mathscr{M}_{k}(\Omega)$ the set of all $k \times k$ matrices whose all eigenvalues lie in $\Omega$.

2010 Mathematics Subject Classification. Primary 47A60, 26E10; Secondary 47A56.

Key words and phrases. Diagonalizable matrix; functional calculus; divided difference; smooth function; scalar operator.

The author gratefully acknowledges the assistance of the Polish Ministry of Sciences and Higher Education grant NN201 546438 for the years 2010-2013. 
Additionally, put

$$
\mathscr{Z}_{k}=\left\{X \in \mathscr{M}_{k}(\mathbb{C}):\left(X-\lambda I_{k}\right)^{k-1} \neq 0=\left(X-\lambda I_{k}\right)^{k} \text { for some } \lambda \in \mathbb{C}\right\}
$$

(where $I_{k}$ stands for the $k \times k$ unit matrix). Two of our main results read as follows:

1.2. Theorem. Let $k \geqslant 3$ be fixed.

(A) Let $\Omega$ be an open set in $\mathbb{C}$ and $f: \Omega \rightarrow \mathbb{C}$ be any function. The following conditions are equivalent:

(i) $f_{\text {op }}: \mathscr{D}_{k}(\Omega) \rightarrow \mathscr{D}_{k}(\mathbb{C})$ is continuous;

(ii) $f_{\text {op }}: \mathscr{D}_{2}(\Omega) \rightarrow \mathscr{D}_{2}(\mathbb{C})$ extends to a continuous function of $\mathscr{M}_{2}(\Omega)$ into $\mathscr{M}_{2}(\mathbb{C})$;

(iii) $f$ is holomorphic.

(B) Let $\Omega$ be a subinterval of $\mathbb{R}$ or an open set in $\mathbb{R}$, and let $f: \Omega \rightarrow \mathbb{C}$ be arbitrary. The following conditions are equivalent:

(i) $f_{\text {op }}: \mathscr{D}_{k}(\Omega) \rightarrow \mathscr{D}_{k}(\mathbb{C})$ is continuous;

(ii) $f_{\text {op }}: \mathscr{D}_{k}(\Omega) \rightarrow \mathscr{D}_{k}(\mathbb{C})$ extends to a continuous function of $\mathscr{M}_{k}(\Omega) \backslash \mathscr{Z}_{k}$ into $\mathscr{M}_{k}(\mathbb{C})$;

(iii) $f$ is of class $C^{k-2}$ and $f^{(k-2)}$ is locally Lipschitz.

Moreover, $f_{\text {op }}$ extends to a continuous function of $\mathscr{M}_{k}(\Omega)$ into $\mathscr{M}_{k}(\mathbb{C})$ iff $f$ is of class $C^{k-1}$. In particular, $f_{\text {op }}: \mathscr{D}_{n}(\Omega) \rightarrow \mathscr{D}_{n}(\mathbb{C})$ is continuous for each $n \geqslant 1$ iff $f$ is of class $C^{\infty}$, and then $f_{\text {op }}$ extends to a continuous function of $\mathscr{M}_{n}(\Omega)$ to $\mathscr{M}_{n}(\mathbb{C})$ for all $n$.

1.3. Theorem. Let $\Omega \subset \mathbb{C}$ and $f: \Omega \rightarrow \mathbb{C}$ be arbitrary. The following conditions are equivalent:

(i) for any $\lambda \in \Omega$ there are positive real constants $M=M(\lambda)$ and $\varepsilon=\varepsilon(\lambda)$ such that $\|f[X]\| \leqslant M$ whenever $\left\|X-\lambda I_{n}\right\| \leqslant \varepsilon, X \in \mathscr{D}_{n}(\Omega)$ and each $n$;

(ii) for any $\lambda \in \Omega$ and each $\varepsilon>0$ there exists $\delta>0$ such that $\left\|f[X]-f\left[\lambda I_{n}\right]\right\| \leqslant \varepsilon$ whenever $\left\|X-\lambda I_{n}\right\| \leqslant \delta, X \in \mathscr{D}_{n}(\Omega)$ and $n$ is arbitrary;

(iii) $f$ extends to a holomorphic function of an open superset of $\Omega$ into $\mathbb{C}$.

Theorem 1.3 shall be applied to characterize those functions $f$ for which $f_{\text {op }}$, as a function acting on (infinite-dimensional) diagonalizable (or so-called scalar) operators, is continuous (see Proposition 6.4 below).

The concept of operator Hölder or operator Lipschitz functions may simply be adapted to the context of functional calculus for diagonalizable matrices (while, in the opposite, operator monotonicity makes no longer sense in this realm). Much weaker property in this direction is uniform continuity. It turns out that for diagonalizable matrices the property of 'matrix uniform continuity' becomes trivial, as shown by

1.4. Proposition. Let $\Omega \subset \mathbb{C}, f: \Omega \rightarrow \mathbb{C}$ and $k \geqslant 3$ be arbitrary (or $k=2$ and $\Omega$ has a cluster point in $\mathbb{C})$. The following conditions are equivalent:

(i) there are positive real constants $M$ and $\varepsilon$ such that $\|f[X]-f[Y]\| \leqslant M$ whenever $X, Y \in \mathscr{D}_{k}(\Omega)$ are such that $\|X-Y\| \leqslant \varepsilon$;

(ii) $f_{\text {op }}: \mathscr{D}_{k}(\Omega) \rightarrow \mathscr{D}_{k}(\mathbb{C})$ is uniformly continuous;

(iii) $f$ is of the form $f(z)=a z+b$ for some $a, b \in \mathbb{C}$.

Theorem 1.3 and the results on the functional calculus for bounded diagonalizable Hilbert space operators presented in Section 6 assert that the classical holomorphic functional calculus for single Banach algebra elements (see e.g. [3, I. $\$ 7]$ ) is as rich as possible even for diagonalizable Hilbert space operators when we require its continuity.

The paper is organized as follows. In Section 2 we present the concept of Tdifferentiability (in the complex sense) of functions defined on arbitrary subsets 
of the complex plane. The idea is based on the Taylor expansion of holomorphic functions. For functions sufficiently many times T-differentiable we define their (in a sense artificial) calculus for matrices, whose 'naturalness' will later be justified by its continuity in case of more 'regular' functions. In the next section we deal with the divided differences for arbitrary functions. We give there certain criteria for the differentiability of a function by means of its divided differences. These results will find applications in the next part, where we give a full characterization of those functions $f: \Omega \rightarrow \mathbb{C}$ for which $f_{\text {op }}: \mathscr{D}_{k}(\Omega) \rightarrow \mathscr{D}_{k}(\mathbb{C})$ is continuous. This section contains also the proof of Theorem 1.2. Section 5 is devoted to the aspects of uniform continuity of $f_{\text {op }}$ and contains the proofs of Theorem 1.3 and Proposition 1.4, while the last part (Section 6) discusses infinite-dimensional case.

Notation and terminology. Whenever $\lambda_{1}, \ldots, \lambda_{n}$ are arbitrary complex numbers, $\operatorname{Diag}\left(\lambda_{1}, \ldots, \lambda_{n}\right)$ denotes the diagonal $n \times n$ matrix whose diagonal entries are precisely $\lambda_{1}, \ldots, \lambda_{n}$. For a complex $n \times n$ matrix $A,\|A\|$ stands for the operator norm of $A=\left[a_{j k}\right]$ induced by the standard inner product on $\mathbb{C}^{n}$, that is:

$$
\|A\|=\max \left\{\left|\sum_{j, k=1}^{n} a_{j k} z_{k} \bar{w}_{j}\right|: z_{1}, w_{1}, \ldots, z_{n}, w_{n} \in \mathbb{C}, \sum_{k=1}^{n}\left|z_{k}\right|^{2}=\sum_{j=1}^{n}\left|w_{j}\right|^{2}=1\right\} .
$$

The matrix $A$ is diagonalizable iff it is similar to a diagonal one, i.e. if $P A P^{-1}$ is diagonal for some invertible $n \times n$ (complex) matrix $P$. The set of all complex eigenvalues of $A$ (the spectrum of $A$ ) is denoted by $\operatorname{sp}(A)$. We denote by $\mu_{A}$ the minimal polynomial for $A$; that is, $\mu_{A}$ is a monic polynomial of a minimal degree such that $\mu_{A}[A]=0$.

Everywhere in this paper $\Omega$ denotes a totally arbitrary nonempty subset of the complex plane and $f$ is any function of $\Omega$ into $\mathbb{C} ; \bar{\Omega}$ and $\Omega^{\prime}(\subset \Omega)$ denote, respectively, the closure of $\Omega$ in $\mathbb{C}$ and the set of all cluster points of $\Omega$, that is, $\Omega^{\prime}$ consists of all $z \in \Omega$ such that $z$ belongs to the closure of $\Omega \backslash\{z\}$. For simplicity, the notation $w \stackrel{\Omega}{\rightarrow} z$ will mean that $w$ runs over $\Omega \backslash\{z\}$ and tends to $z$. A complex-valued function defined on a subset of $\mathbb{C}^{n}$ is said to be locally Lipschitz iff every point of its domain has a relative neighbourhood (i.e. relatively open in the domain of the function) on which the function is Lipschitz. Subintervals of the real line are assumed to be nondegenerate.

For any $k \geqslant 1$, let us denote by $\mathscr{M}_{k}(\Omega)$ and $\mathscr{D}_{k}(\Omega)$, respectively, the sets of all complex $k \times k$ matrices $X$ with $\operatorname{sp}(X) \subset \Omega$ and all such diagonalizable matrices. Observe that:

(M1) $\mathscr{D}_{k}(\Omega)=\left\{X \in \mathscr{M}_{k}(\Omega)\right.$ : all roots of $\mu_{X}$ are simple $\}$.

Further, we put:

$(\mathrm{M} 2) \mathscr{M}_{k}^{o}(\Omega)=\left\{X \in \mathscr{M}_{k}(\Omega)\right.$ : all possible multiple roots of $\mu_{X}$ belong to $\left.\Omega^{\prime}\right\}$.

\section{Abstract CONCEPt of Differentiability}

The idea of the Taylor expansion enables us to introduce the following

2.1. Definition. Let $k \geqslant 1$. A function $f: \Omega \rightarrow \mathbb{C}$ is said to be $k$ times $T$ differentiable at a point $a \in \Omega^{\prime}$ (the prefix ' $\mathrm{T}$-' is to emphasize the role of the Taylor expansion) iff there are complex numbers $u_{0}, \ldots, u_{k}$ and a function $\tau_{a}: \Omega \rightarrow \mathbb{C}$ such that

$$
\begin{aligned}
& \text { - } f(z)=\sum_{j=0}^{k} \frac{u_{j}}{j !}(z-a)^{j}+\tau_{a}(z)(z-a)^{k} \quad(z \in \Omega) \text {, } \\
& \text { - } \tau_{a}(a)=0 \text {, } \\
& \text { - } \tau_{a} \text { is continuous at } a .
\end{aligned}
$$


It is easy to observe that the numbers $u_{0}, \ldots, u_{k}$ and the function $\tau_{a}$ are uniquely determined by (2-1) and thus we may put $f^{(j)}(a):=u_{j}(j=0, \ldots, k)$ whenever $(2-1)$ holds. (In particular, $f^{(0)}(a)=f(a)$.) We say $f$ is $k$ times T-differentiable iff it is so at each point of $\Omega^{\prime}$. In that case we call the function $f^{(j)}: \Omega^{\prime} \rightarrow \mathbb{C}$ the $j$ th $T$-derivative of $f$. Finally, $f$ is said to be of class TC $^{k}$ if $f$ is $k$ times T-differentiable and the functions $f^{\prime}, \ldots, f^{(k)}: \Omega^{\prime} \rightarrow \mathbb{C}$ as well as

$$
\tau: \Omega^{\prime} \times \Omega \ni(a, x) \mapsto \tau_{a}(x) \in \mathbb{C}
$$

(where $\tau_{a}$ is as in (2-1)) are continuous. When $f$ is of class $\mathrm{TC}^{k}$ for each $k$, we express this by writing that $f$ is of class $\mathrm{TC}^{\infty}$. Additionally, we call the function $f$ of class $\mathrm{TC}^{0}$ if it is continuous, and we identify $f^{(0)}$ with $f$ (so, the domain of $f^{(0)}$ coincides with $\Omega$, which may differ from $\Omega^{\prime}$ ).

The reader should notice that if $f$ is T-differentiable, then $f$ is continuous and $f^{\prime}(z)=\lim _{w \rightarrow z} \frac{f(w)-f(z)}{w-z}$ for each $z \in \Omega^{\prime}$. In particular, if $\Omega$ is open in $\mathbb{C}$, then $f$ is $T$-differentiable iff it is of class $\mathrm{TC}^{\infty}$, iff it is holomorhic. Much more difficult in proving is the following result, due to Whitney [14].

2.2. Proposition. If $\Omega \subset \mathbb{R}$ is a subinterval or an open subset of the real line, then for arbitrary $k \in\{1,2, \ldots\}$ a function $f: \Omega \rightarrow \mathbb{C}$ is of class TC $^{k}$ iff it is of class $C^{k}$.

Proof. Sufficiency follows from Taylor's theorem, while necessity is a consequence of [14, Theorem 3] (indeed, due to that result, a function is of class $C^{k}$ provided $\tau_{a}(x)$ tends to 0 when $x$ tends to $a$ and this convergence is uniform on compact sets).

We have introduced T-differentiable functions in order to extend the classical (polynomial or holomorphic) functional calculus for matrices as widely as possible. First of all note that if $X$ is a square matrix with $\mu_{X}(z)=\prod_{j=1}^{m}\left(z-\lambda_{j}\right)^{p_{j}}$ (where $\lambda_{j}$ 's are different) and $P$ and $Q$ are two arbitrary (complex) polynomials such that $P^{(s)}\left(\lambda_{j}\right)=Q^{(s)}\left(\lambda_{j}\right)$ for any $j \in\{1, \ldots, m\}$ and $s \in\left\{0, \ldots, p_{j}-1\right\}$, then $P[X]=Q[X]$. This simple observation leads us to the following

2.3. Definition. Let $f: \Omega \rightarrow \mathbb{C}$ be of class $\operatorname{TC}^{k}$ (where $k \in\{0,1,2, \ldots, \infty\}$ ). Let $X \in \mathscr{M}_{n}^{o}(\Omega)($ cf. $(\mathrm{M} 2))$ be a matrix such that $\mu_{X}(z)=\prod_{j=1}^{m}\left(z-\lambda_{j}\right)^{p_{j}}$ (where $\lambda_{j}$ 's are different) and $p_{j} \leqslant k+1$ for each $j$. Let $P$ be a polynomial such that for each $j \in\{1, \ldots, m\}$ :

- $P^{(s)}\left(\lambda_{j}\right)=f^{(s)}\left(\lambda_{j}\right)$ for $s \in\left\{0, \ldots, p_{j}-1\right\}$ provided $\lambda_{j} \in \Omega^{\prime}$;

- $P\left(\lambda_{j}\right)=f\left(\lambda_{j}\right)$ provided $\lambda_{j} \notin \Omega^{\prime}$.

We define the matrix $f[X]$ as $P[X]$. The note preceding the definition shows that $f[X]$ is well defined - that is, it is independent of the choice of $P$ (recall that $p_{j}=1$ if $\lambda_{j} \notin \Omega^{\prime}$, since $X \in \mathscr{M}_{n}^{o}(\Omega)$ ). In particular, in this way we obtain functions

$$
\begin{aligned}
& f_{\text {op }}: \mathscr{M}_{n}^{o}(\Omega) \ni X \mapsto f[X] \in \mathscr{M}_{n}(\mathbb{C}) \quad(0<n<k+2), \\
& f_{\text {op }}: \mathscr{M}_{n}^{o}(\Omega) \backslash \mathscr{Z}_{n} \ni X \mapsto f[X] \in \mathscr{M}_{n}(\mathbb{C}) \quad(n=k+2)
\end{aligned}
$$

(where $\mathscr{Z}_{n}$ is as in (1-1)). We call the assignment $f \mapsto f_{\text {op }}$ the extended functional calculus for matrices.

Although the extended functional calculus strikely resembles holomorphic, there is no algebraic nor 'practical' justification (apart from the calculus for diagonalizable matrices) of the approach introduced above. Since $\mathscr{D}_{n}(\Omega)$ is dense in $\mathscr{M}_{n}^{o}(\Omega)$ (to convince of that, use e.g. Jordan's matrix decomposition theorem) and $f_{\text {op }}$ is naturally defined on $\mathscr{D}_{n}(\Omega)$ (for totally arbitrary functions $f: \Omega \rightarrow \mathbb{C}$ ), thus the continuity of $f_{\text {op }}$ (on $\mathscr{M}_{n}^{o}(\Omega)$ ) would be a sufficiently good justification. In the 
course of our research on this issue, it turned out that if only $f_{\text {op }}$ is continuous on $\mathscr{D}_{n}(\Omega)$ (where $n>1$ ), then $f$ is of class $\mathrm{TC}^{n-2}$ and $f_{\text {op }}$ (defined as above) is automatically continuous on $\mathscr{M}_{n}^{o}(\Omega) \backslash \mathscr{Z}_{n}$ as well as on $\mathscr{M}_{n-1}^{o}(\Omega)$. These two results motivated us to introduce Definition 2.3. However, it is worth noting that being of class TC $^{\infty}$ for a function $f$ is insufficient for $f_{\text {op }}$ to be continuous on $\mathscr{D}_{2}(\Omega)$ in general (see Example 4.6 below). To hit the mark of the problem, a stronger notion of differentiability is needed, which we now turn to.

\section{DIVIDED DIFFERENCES}

We begin this part with recalling the concept of divided differences and their basic properties. For each $n \geqslant 1$ let $\Omega^{(n)}$ be the set of all vectors $\left(z_{1}, \ldots, z_{n}\right) \in \Omega^{n}$ whose all coordinates are different. Further, let $\Omega^{[n]}$ stand for the set of all vectors $\left(z_{1}, \ldots, z_{n}\right) \in \Omega^{n}$ satisfying the following condition: if $z_{j}=z_{k}$ for some distinct $j$ and $k$, then $z_{j} \in \Omega^{\prime}$. Notice that the closure of $\Omega^{(n)}$ (in $\mathbb{C}^{n}$ ) coincides with $\bar{\Omega}^{[n]}$. The divided difference of a function $f: \Omega \rightarrow \mathbb{C}$ at $\left(z_{1}, \ldots, z_{n}\right) \in \Omega^{(n)}$, denoted by $\Delta\left(z_{1}, \ldots, z_{n}\right) f$, is defined by induction on $n \geqslant 1$ as follows:

- $\Delta(z) f=f(z)$ for any $z \in \Omega^{(1)}(=\Omega)$;

- $\Delta\left(z_{1}, \ldots, z_{n}\right) f=\left[\Delta\left(z_{2}, \ldots, z_{n}\right) f-\Delta\left(z_{1}, \ldots, z_{n-1}\right) f\right] /\left(z_{n}-z_{1}\right)$ for $n>1$.

Let us now list two most important for us properties of the divided differences:

(DD1) The divided differences are symmetric; that is, whenever $\left(z_{1}, \ldots, z_{n}\right) \in$ $\Omega^{(n)}$ and $\sigma$ is a permutation of $\{1, \ldots, n\}$, then $\Delta\left(z_{\sigma(1)}, \ldots, z_{\sigma(n)}\right) f=$ $\Delta\left(z_{1}, \ldots, z_{n}\right) f$.

(DD2) For any $\left(z_{1}, \ldots, z_{n}\right) \in \Omega^{(n)}$ (where $\left.n>1\right)$, the polynomial $W(z):=f\left(z_{1}\right)+$ $\sum_{k=2}^{n} \Delta\left(z_{1}, \ldots, z_{k}\right) f \cdot \prod_{j=1}^{k-1}\left(z-z_{j}\right)$ satisfies the equations $W\left(z_{j}\right)=f\left(z_{j}\right)$ for $j=1, \ldots, n$.

For the proofs of the above facts and a more detailed discussion on divided differences, consult [4].

Our interest are functions whose divided differences satisfy some additional conditions. To this end, we introduce

3.1. Definition. Let $k \in\{0,1,2, \ldots\}$. A function $f: \Omega \rightarrow \mathbb{C}$ is said to be of class $\mathrm{DDB}^{k}$, in symbols $f \in \mathrm{DDB}^{k}(\Omega)$ ['DD' and 'B' are the first letters of 'divided differences' and 'bounded'], if for any $z \in \Omega^{\prime}$ there are positive real constants $M=$ $M(\lambda)$ and $\varepsilon=\varepsilon(\lambda)$ such that $\left|\Delta\left(z_{1}, \ldots, z_{k+1}\right) f\right| \leqslant M$ whenever $\left(z_{1}, \ldots, z_{k+1}\right) \in$ $\Omega^{(k+1)}$ and $\left|z_{j}-z\right| \leqslant \varepsilon$. The function $f$ is of class $\operatorname{DDB}^{\infty}\left(f \in \operatorname{DDB}^{\infty}(\Omega)\right)$ if it is of class DDB ${ }^{k}$ for any $k$.

Similarly, $f$ is said to be of class $\operatorname{DDC}^{k}$, in symbols $f \in \operatorname{DDC}^{k}(\Omega)$ ['C' is the first letter of 'continuous'], if the function $\Omega^{(k+1)} \ni\left(z_{1}, \ldots, z_{k+1}\right) \mapsto \Delta\left(z_{1}, \ldots, z_{k+1}\right) f \in$ $\mathbb{C}$ has (finite) limit at $(z, \ldots, z) \in \mathbb{C}^{k+1}$ for each $z \in \Omega^{\prime}$. Finally, $f$ is of class DDC ${ }^{\infty}$ $\left(f \in \operatorname{DDC}^{\infty}(\Omega)\right)$ if $f \in \operatorname{DDC}^{k}(\Omega)$ for each $k$.

It follows from the very definitions that $\operatorname{DDC}^{k}(\Omega) \subset \operatorname{DDB}^{k}(\Omega)$ for any $k$. At first sight, it may seem that the classes DDB and DDC have not much more in common (for example, $f \in \operatorname{DDB}^{0}(\Omega)$ iff $f$ is locally bounded, while $f \in \operatorname{DDC}^{0}(\Omega)$ iff $f$ is continuous). Therefore the following result may be surprising.

3.2. Proposition. For any $n \in\{1,2,3, \ldots\}, \operatorname{DDB}^{n}(\Omega) \subset \operatorname{DDC}^{n-1}(\Omega)$. Moreover, if $f \in \operatorname{DDB}^{n}(\Omega)$, then there exists a locally compact set $G, \Omega \subset G \subset \bar{\Omega}$, such that for any $k \in\{1, \ldots, n\}$ the function $\Omega^{(k)} \ni\left(z_{1}, \ldots, z_{k}\right) \mapsto \Delta\left(z_{1}, \ldots, z_{k}\right) f \in \mathbb{C}$ extends to a locally Lipschitz symmetric function of $G^{[k]}$ into $\mathbb{C}$.

In particular, $\operatorname{DDB}^{\infty}(\Omega)=\operatorname{DDC}^{\infty}(\Omega)$. 
Proof. Clearly, it is enough to prove the claims of the first paragraph of the proposition. We shall do this by induction on $n$. To simplify the argument, for each $n>0$ put

$$
\Theta_{n}=\{(z, \ldots, z): z \in \mathbb{C}\} \subset \mathbb{C}^{n}
$$

When $n=1$, the assumption that $f \in \operatorname{DDB}^{1}(\Omega)$ means that $f$ is locally Lipschitz at each point of $\Omega^{\prime}$. Since the other points of $\Omega$ are isolated, we infer that $f$ is locally Lipschitz. So, for each $z \in \Omega$ there are positive real constants $\varepsilon_{z}$ and $M_{z}$ such that $\left|f(w)-f\left(w^{\prime}\right)\right| \leqslant M_{z}\left|w-w^{\prime}\right|$ for any $w, w^{\prime} \in \Omega \cap B\left(z, \varepsilon_{z}\right)$ where

$$
B\left(z, \varepsilon_{z}\right)=\left\{w \in \mathbb{C}:|w-z|<\varepsilon_{z}\right\} .
$$

We infer from the completeness of $\mathbb{C}$ that the restriction of $f$ to $\Omega \cap B\left(z, \varepsilon_{z}\right)$ extends to a Lipschitz function $g_{z}: \bar{\Omega} \cap B\left(z, \varepsilon_{z}\right) \rightarrow \mathbb{C}$. It is readily seen that the functions $g_{z}$ 's $(z \in \Omega)$ agree and hence their union is locally Lipschitz at each point of its domain $G:=\bar{\Omega} \cap \bigcup_{z \in \Omega} B\left(z, \varepsilon_{z}\right)$. It remains to note that $G$ is locally compact as the intersection of an open and a closed set.

Now assume the assertion holds for $n-1$ (where $n>1$ ); take $f \in \operatorname{DDB}^{n}(\Omega)$ and fix $z \in \Omega^{\prime}$. Let $\varepsilon_{z}>0$ and $M_{z}>0$ be such that $\left|\Delta\left(z_{1}, \ldots, z_{n+1}\right) f\right| \leqslant M_{z}$ whenever $z_{1}, \ldots, z_{n+1} \in \Omega \cap B\left(z, \varepsilon_{z}\right)$ are different. This means that

$$
\left|\Delta\left(z_{1}, \ldots, z_{n}\right) f-\Delta\left(z_{2}, \ldots, z_{n+1}\right) f\right| \leqslant M_{z}\left|z_{1}-z_{n+1}\right|
$$

for $\left(z_{1}, \ldots, z_{n+1}\right) \in \Omega^{(n+1)} \cap\left[B\left(z, \varepsilon_{z}\right)\right]^{n+1}$. Let us now show that

$$
\left|\Delta\left(z_{1}, \ldots, z_{n}\right) f-\Delta\left(w_{1}, \ldots, w_{n}\right) f\right| \leqslant M_{z} \sum_{j=1}^{n}\left|z_{j}-w_{j}\right|
$$

for any $\left(z_{1}, \ldots, z_{n}\right),\left(w_{1}, \ldots, w_{n}\right) \in \Omega^{(n)} \cap\left[B\left(z, \varepsilon_{z}\right)\right]^{n}$. To this end, put $I:=$ $\left\{z_{1}, \ldots, z_{n}\right\} \cap\left\{w_{1}, \ldots, w_{n}\right\}$. Involving (DD1) and permuting both the systems $\left(z_{1}, \ldots, z_{n}\right)$ and $\left(w_{1}, \ldots, w_{n}\right)$ by means of a common permutation, we may and do assume that $I=\left\{z_{j}: j \leqslant k\right\}$ for some $k \leqslant n$ ( $k$ may be equal to 0$)$. If $k=n$, we are done thanks to (DD1). Hence, we may and do assume that $k<n$. For $s \in\{1, \ldots, k\}$ denote by $\sigma(s)$ a unique index $j$ for which $w_{j}=z_{s}$. Now fix for a moment $s \in\{k+1, \ldots, n\}$ and put $\nu_{s}(1)=s$. Further we make use of induction: assume $\nu_{s}(j)$ is already defined for some $j \geqslant 1$. If $w_{\nu_{s}(j)} \in I$, define $\nu_{s}(j+1)$ as a unique index $j^{\prime}$ for which $w_{\nu_{s}(j)}=z_{j^{\prime}}$. Otherwise put $m(s)=j$, $\sigma(s)=\nu_{s}(j)$ and finish the construction for $s$. In this way we obtain a sequence $z_{s}, w_{\nu_{s}(1)}, \ldots, w_{\nu_{s}(m(s))}$ such that $w_{\nu_{s}(m(s))}=w_{\sigma(s)} \notin\left\{z_{1}, \ldots, z_{n}\right\}$ and

$(\bullet) w_{\nu_{s}(j)}=z_{\nu_{s}(j+1)} \in I$ for $0 \leqslant j<m(s)$ with convention that $w_{\nu_{s}(0)}:=z_{s}$.

The above construction shows also that

$(\bullet \bullet) \nu_{s}(j) \neq \nu_{s^{\prime}}\left(j^{\prime}\right)$ provided $s, s^{\prime}>k, 0<j \leqslant m(s), 0<j^{\prime} \leqslant m\left(s^{\prime}\right)$ and $(s, j) \neq\left(s^{\prime}, j^{\prime}\right)$

and $\sigma:\{1, \ldots, n\} \rightarrow\{1, \ldots, n\}$ is a permutation. Now (DD1) yields that

$$
\begin{gathered}
\left|\Delta\left(z_{1}, \ldots, z_{n}\right) f-\Delta\left(w_{1}, \ldots, w_{n}\right) f\right|=\left|\Delta\left(z_{1}, \ldots, z_{n}\right) f-\Delta\left(w_{\sigma(1)}, \ldots, w_{\sigma(n)}\right) f\right|= \\
=\left|\Delta\left(z_{1}, \ldots, z_{k}, z_{k+1}, \ldots, z_{n}\right) f-\Delta\left(z_{1}, \ldots, z_{k}, w_{\sigma(k+1)}, \ldots, w_{\sigma(n)}\right) f\right| \leqslant \\
\leqslant \sum_{s=k+1}^{n}\left|\Delta\left(z_{1}, \ldots, z_{s}, w_{\sigma(s+1)}, \ldots, w_{\sigma(n)}\right) f-\Delta\left(z_{1}, \ldots, z_{s-1}, w_{\sigma(s)}, \ldots, w_{\sigma(n)}\right) f\right| .
\end{gathered}
$$


Thanks to (3-3) (and again (DD1)), we may continue the above estimations as follows (see the convention in $(\bullet)$ ):

$$
\begin{gathered}
\left|\Delta\left(z_{1}, \ldots, z_{n}\right) f-\Delta\left(w_{1}, \ldots, w_{n}\right) f\right| \leqslant M_{z} \sum_{s=k+1}^{n}\left|z_{s}-w_{\sigma(s)}\right|= \\
=M_{z} \sum_{s=k+1}^{n}\left|z_{s}-w_{\nu_{s}\left(m_{s}\right)}\right| \leqslant M_{z} \sum_{s=k+1}^{n} \sum_{j=1}^{m(s)}\left|w_{\nu_{s}(j-1)}-w_{\nu_{s}(j)}\right| \leqslant \\
\leqslant M_{z} \sum_{s=k+1}^{n} \sum_{j=1}^{m(s)}\left|z_{\nu_{s}(j)}-w_{\nu_{s}(j)}\right| \leqslant M_{z} \sum_{j=1}^{n}\left|z_{j}-w_{j}\right|
\end{gathered}
$$

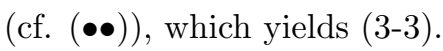

The inequality (3-4) implies that there is a Lipschitz function

$$
g_{z}: \bar{\Omega}^{[n]} \cap\left[B\left(z, \varepsilon_{z}\right)\right]^{n} \rightarrow \mathbb{C}
$$

such that

$$
g_{z}\left(z_{1}, \ldots, z_{n}\right)=\Delta\left(z_{1}, \ldots, z_{n}\right) f
$$

for $\left(z_{1}, \ldots, z_{n}\right) \in \Omega^{(n)} \cap\left[B\left(z, \varepsilon_{z}\right)\right]^{n}$. The density argument combined with (DD1) yields the symmetry of $g_{z}$.

The above argument shows that $f \in \operatorname{DDC}^{n-1}(\Omega)\left(\subset \operatorname{DDB}^{n-1}(\Omega)\right)$. So, it follows from the induction hypothesis that there are a locally compact set $G_{0}\left(\Omega \subset G_{0} \subset \bar{\Omega}\right)$ and locally Lipschitz symmetric functions $F_{k}: G_{0}^{[k]} \rightarrow \mathbb{C}(k=1, \ldots, n-1)$ such that

$$
F_{k}\left(z_{1}, \ldots, z_{k}\right)=\Delta\left(z_{1}, \ldots, z_{k}\right) f
$$

whenever $\left(z_{1}, \ldots, z_{k}\right) \in \Omega^{(k)}$. For simplicity, we introduce the following notation: for any $z=\left(z_{1}, \ldots, z_{n}\right) \in \mathbb{C}^{n}$ and $j \in\{1, \ldots, n\}$, let $z_{j}^{\prime}$ be the vector in $\mathbb{C}^{n-1}$ which is obtained from $z$ by erasing its $j$ th coordinate. Observe that for any $j, k, l, m \in\{1, \ldots, n\}$ the set $A:=\left\{z=\left(z_{1}, \ldots, z_{n}\right) \in G_{0}^{[n]}:\left(F_{n-1}\left(z_{j}^{\prime}\right)-\right.\right.$ $\left.\left.F_{n-1}\left(z_{k}^{\prime}\right)\right)\left(z_{m}-z_{l}\right)=\left(F_{n-1}\left(z_{l}^{\prime}\right)-F_{n-1}\left(z_{m}^{\prime}\right)\right)\left(z_{k}-z_{j}\right)\right\}$ is relatively closed in $G_{0}^{[n]}$. What is more, we deduce from (DD1) and (3-6) that $A \supset \Omega^{(n)}$. (Note that if $j \neq k$ and $\left(z_{1}, \ldots, z_{n}\right) \in \Omega^{(n)}$, then $\left[F_{n-1}\left(z_{j}^{\prime}\right)-F_{n-1}\left(z_{k}^{\prime}\right)\right] /\left(z_{k}-z_{j}\right)=\left[\Delta\left(z_{j}^{\prime}\right) f-\right.$ $\left.\Delta\left(z_{k}^{\prime}\right) f\right] /\left(z_{k}-z_{j}\right)=\Delta\left(z_{1}, \ldots, z_{n}\right) f$.) So, the density of $\Omega^{(n)}$ in $G_{0}^{[n]}$ implies that $A=G_{0}^{[n]}$. We conclude that if $\left(z_{1}, \ldots, z_{n}\right) \in G_{0}^{[n]}$ and $j, k, l, m \in\{1, \ldots, n\}$ are such that $z_{j} \neq z_{k}$ and $z_{l} \neq z_{m}$, then $\frac{F_{n-1}\left(z_{j}^{\prime}\right)-F_{n-1}\left(z_{k}^{\prime}\right)}{z_{k}-z_{j}}=\frac{F_{n-1}\left(z_{l}^{\prime}\right)-F_{n-1}\left(z_{m}^{\prime}\right)}{z_{m}-z_{l}}$. The above property enables us to define properly a function $F: G_{0}^{[n]} \backslash \Theta_{n} \rightarrow \mathbb{C}$ (see (3-1) for the definition of $\Theta_{n}$ ) by the rule:

$$
F\left(z_{1}, \ldots, z_{n}\right)=\frac{F_{n-1}\left(z_{j}^{\prime}\right)-F_{n-1}\left(z_{k}^{\prime}\right)}{z_{k}-z_{j}}
$$

where $j$ and $k$ are chosen so that $z_{j} \neq z_{k}$. F is locally Lipschitz-since locally it is the quotient of two locally Lipschitz functions. And $F$ is symmetric since $F_{n-1}$ is such. Finally,

$$
F\left(z_{1}, \ldots, z_{n}\right)=\Delta\left(z_{1}, \ldots, z_{n}\right) f \quad\left(\left(z_{1}, \ldots, z_{n}\right) \in \Omega^{(n)}\right)
$$

which follows from $(3-6)$.

To end the proof, observe that the functions $F$ and $g_{z}$ 's $\left(z \in \Omega^{\prime}\right)$ agree. Hence it suffices to define $G$ as the intersection of $G_{0}$ and $\bar{\Omega} \cap \bigcup_{z \in \Omega^{\prime}} B\left(z, \varepsilon_{z}\right)$ and the extension of $\Omega^{(n)} \ni\left(z_{1}, \ldots, z_{n}\right) \mapsto \Delta\left(z_{1}, \ldots, z_{n}\right) \in \mathbb{C}$ we search for as the union of $F$ and $g_{z}$ 's (understood as a function on $G^{[n]}$ ). 
Since the proof of the next result is similar to the above, we skip it.

3.3. Proposition. If $f \in \operatorname{DDC}^{n}(\Omega)$, then for any $k \in\{1, \ldots, n+1\}$ the function $\Omega^{(k)} \ni\left(z_{1}, \ldots, z_{k}\right) \rightarrow \Delta\left(z_{1}, \ldots, z_{k}\right) f \in \mathbb{C}$ extends to a continuous symmetric function $F_{k}: \Omega^{[k]} \rightarrow \mathbb{C}$.

It is an easy exercise that a holomorphic function is of class DDC ${ }^{\infty}$ (this immediately follows from Theorem 4.3 below). Divided differences are also involved in the characterization of one real variable functions extendable to functions of class $C^{k}$ given by Whitney [15]:

3.4. Theorem. Let $\Omega \subset \mathbb{R}$ be a closed set and $k \geqslant 0$. A function $f: \Omega \rightarrow \mathbb{C}$ extends to a function of class $C^{k}$ of $\mathbb{R}$ into $\mathbb{C}$ iff $f \in \operatorname{DDC}^{k}(\Omega)$.

Now we prove a generalization of the easier part of the above result.

3.5. Proposition. Each function of class $\mathrm{DDC}^{k}$ is of class $\mathrm{TC}^{k}$. Moreover, if $f \in$ $\operatorname{DDC}^{k}(\Omega)$ and the functions $F_{j}: \Omega^{[j]} \rightarrow \mathbb{C}(j=1, \ldots, k)$ are as in Proposition 3.3, then $f^{(j-1)}(z)=(j-1) ! \cdot F_{j}(z, \ldots, z)$ for any $j \in\{1, \ldots, k+1\}$ and $z \in \Omega^{\prime}$.

Proof. Fix $z \in \Omega^{\prime}$, take arbitrary $w \in \Omega$ and let $z_{1}, \ldots, z_{k} \in \Omega \backslash\{w\}$ be distinct points. Put

$$
W(z)=\sum_{j=1}^{k} F_{j}\left(z_{1}, \ldots, z_{j}\right) \prod_{s=1}^{j-1}\left(z-z_{s}\right)+F_{k+1}\left(z_{1}, \ldots, z_{k}, w\right) \prod_{s=1}^{k}\left(z-z_{s}\right) .
$$

It follows from (DD2) that $W(w)=f(w)$, from which we deduce that

$$
f(w)=\sum_{j=1}^{k} F_{j}\left(z_{1}, \ldots, z_{j}\right) \prod_{s=1}^{j-1}\left(w-z_{s}\right)+F_{k+1}\left(z_{1}, \ldots, z_{k}, w\right) \prod_{s=1}^{k}\left(w-z_{s}\right) .
$$

Now if $z_{j} \rightarrow z$ for $j \in\{1, \ldots, k\},(3-8)$ changes into (thanks to the continuity of $\left.F_{1}, \ldots, F_{k+1}\right)$ :

$$
\begin{aligned}
& f(w)=\sum_{j=1}^{k} F_{j}(z, \ldots, z)(w-z)^{j-1}+F_{k+1}(z, \ldots, z, w)(w-z)^{k} \\
& =\sum_{j=1}^{k+1} F_{j}(z, \ldots, z)(w-z)^{j-1}+\left[F_{k+1}(z, \ldots, z, w)-F_{k+1}(z, \ldots, z, z)\right](w-z)^{k} .
\end{aligned}
$$

So, to finish the proof it suffices to define $\tau_{z}: \Omega \rightarrow \mathbb{C}$ as $\tau_{z}(w)=F_{k+1}(z, \ldots, z, w)-$ $F_{k+1}(z, \ldots, z, z)$ and note that the function $\Omega^{\prime} \times \Omega \ni(z, w) \mapsto \tau_{z}(w) \in \mathbb{C}$ is continuous.

As a simple consequence of the above result, we obtain

3.6. Corollary. Let $\Omega \subset \mathbb{R}$ be a subinterval or an open subset of the real line and $k \geqslant 1$. A function $f: \Omega \rightarrow \mathbb{C}$ is of class $\mathrm{DDB}^{k}$ iff $f$ is of class $C^{k-1}$ and $f^{(k-1)}$ is locally Lipschitz.

Proof. First assume $f$ is of class $C^{k-1}$ and $f^{(k-1)}$ is locally Lipschitz. Fix $a \in \Omega$ and let $\varepsilon>0$ and $M$ be such that $\left|f^{(k-1)}(x)-f^{(k-1)}(y)\right| \leqslant M|x-y|$ for any $x, y \in \Omega \cap[a-\varepsilon, a+\varepsilon]$ and this last set is an interval. Let $x_{1}, \ldots, x_{k+1}$ be distinct points of $\Omega \cap[a-\varepsilon, a+\varepsilon]$. It suffices to check that $\left|\Delta\left(x_{1}, \ldots, x_{k+1}\right) u\right| \leqslant M$ for $u \in\{\operatorname{Re} f, \operatorname{Im} f\}$. So, we may assume $f$ is real-valued. Moreover, thanks to property (DD1) we may also assume that $x_{1}<\ldots<x_{k+1}$. It follows from the mean value theorem for divided differences (see e.g. the argument on page 369 in [15]) that 
there are $\xi \in\left(x_{1}, x_{k}\right)$ and $\eta \in\left(x_{2}, x_{k+1}\right)$ such that $\Delta\left(x_{1}, \ldots, x_{k}\right) f=f^{(k-1)}(\xi)$ and $\Delta\left(x_{2}, \ldots, x_{k+1}\right) f=f^{(k-1)}(\eta)$. Finally, observe that then

$$
\left|\Delta\left(x_{1}, \ldots, x_{k+1}\right) f\right|=\frac{\left|f^{(k-1)}(\eta)-f^{(k-1)}(\xi)\right|}{x_{k+1}-x_{1}} \leqslant M \cdot \frac{|\eta-\xi|}{x_{k+1}-x_{1}} \leqslant M .
$$

Conversely, if $f \in \operatorname{DDB}^{k}(\Omega)$, then $f \in \operatorname{DDC}^{k-1}(\Omega)$ (by Proposition 3.2) and hence $f$ is of class TC $^{k-1}$ (cf. Proposition 3.5). Consequently, $f$ is of class $C^{k-1}$, by Whitney's theorem (Proposition 2.2). Finally, we infer from Propositions 3.5 and 3.2 that $f^{(k-1)}$ is locally Lipschitz.

3.7. Remark. Using similar arguments as those in the proof of Proposition 3.5, one may check that if a function $f: \Omega \rightarrow \mathbb{C}$ is of class $\operatorname{DDC}^{k}(k=1,2, \ldots, \infty)$, then for any $j<k(j \geqslant 1)$ the function $f^{(j)}: \Omega^{\prime} \rightarrow \mathbb{C}$ is of class $\mathrm{TC}^{k-j}$ and $\left(f^{(j)}\right)^{(s)}=\left.\left(f^{(j+s)}\right)\right|_{\Omega^{\prime \prime}}\left(\right.$ where $\left.\Omega^{\prime \prime}=\left(\Omega^{\prime}\right)^{\prime}\right)$ for $s=1, \ldots, k-j$.

\section{Continuity of functional Calculus}

Let us begin this section with a reminder that for totally arbitrary function $f: \Omega \rightarrow \mathbb{C}$ the function $f_{\text {op }}: \mathscr{D}_{k}(\Omega) \rightarrow \mathscr{D}_{k}(\mathbb{C})$ is well defined for any $k$ by the following rule: $f[D]=W[D]$ where $D$ is a diagonalizable square matrix and $W$ is any polynomial such that $\left.f\right|_{\mathrm{sp}(D)}=\left.W\right|_{\mathrm{sp}(D)}$. We are interested in those functions $f$ for which $f_{\text {op }}$ is continuous on $\mathscr{D}_{k}(\Omega)$ (for fixed $k$ ). Observe that $f_{\text {op }}: \mathscr{D}_{1}(\Omega) \rightarrow \mathscr{M}_{1}(\mathbb{C})$ may naturally be identified with $f: \Omega \rightarrow \mathbb{C}$ and hence for $k=1$ the characterization is trivial $\left(f_{\text {op }}: \mathscr{D}_{1}(\Omega) \rightarrow \mathscr{M}_{1}(\mathbb{C})\right.$ is continuous iff $f$ is such). Therefore everywhere below we will assume that $k>1$.

In what follows, we shall use the following concept, very often practiced. For a $k \times k$ matrix $A$ we write $\operatorname{sp}(A)=\left\{\lambda_{1}, \ldots, \lambda_{k}\right\}$ iff the characteristic polynomial $W_{A}(z)=\operatorname{det}(z I-A)$ of $A$ has the form $W_{A}(z)=\left(z-\lambda_{1}\right) \cdot \ldots \cdot\left(z-\lambda_{k}\right)$. (So, in the unordered $k$-tuple $\left\{\lambda_{1}, \ldots, \lambda_{k}\right\}$ the number of appearances of each of eigenvalues of $A$ coincides with its algebraic multiplicity, i.e. its multiplicity as a root of $W_{A}$.)

The following is a well-known result (see e.g. [13, Theorem 3.1.2]). We will apply it in the proof of Theorem 4.3 below.

4.1. Proposition. If $k \times k$ matrices $A_{1}, A_{2}, A_{3}, \ldots$ converge to a matrix $A$ and $\sigma(A)=\left\{\lambda^{(1)}, \ldots, \lambda^{(k)}\right\}$, then there are scalar sequences $\left(\lambda_{n}^{(1)}\right)_{n=1}^{\infty}, \ldots,\left(\lambda_{n}^{(k)}\right)_{n=1}^{\infty}$ such that $\lim _{n \rightarrow \infty} \lambda_{n}^{(j)}=\lambda^{(j)}$ for $j=1, \ldots, k$ and $\sigma\left(A_{n}\right)=\left\{\lambda_{n}^{(1)}, \ldots, \lambda_{n}^{(k)}\right\}$ for any $n \geqslant 1$.

The proof of next simple result is left to the reader.

4.2. Lemma. The closure of $\mathscr{D}_{k}(\Omega)$ in $\mathscr{M}_{k}(\mathbb{C})$ coincides with $\mathscr{M}_{k}^{o}(\bar{\Omega})$.

The above lemma explains the role played by the set $\mathscr{M}_{k}^{o}(\Omega)$ and shows that this set appears quite naturally in topological aspects.

Now we are ready to state and prove the main result of the section.

4.3. Theorem. For an arbitrary function $f: \Omega \rightarrow \mathbb{C}$ and $k \geqslant 2$ the following conditions are equivalent:

(i) for any $\lambda \in \Omega^{\prime}$ there are positive real numbers $\varepsilon=\varepsilon(\lambda)$ and $M=M(\lambda)$ such that $\|f[X]\| \leqslant M$ provided $X \in \mathscr{D}_{k}(\Omega)$ is such that $\|X-\lambda I\| \leqslant \varepsilon$;

(ii) $f_{\text {op }}: \mathscr{D}_{k}(\Omega) \rightarrow \mathscr{D}_{k}(\mathbb{C})$ is continuous;

(iii) $f$ is of class $\mathrm{TC}^{k-2}$ and $f_{\text {op }}: \mathscr{M}_{k}^{o}(\Omega) \backslash \mathscr{Z}_{k} \rightarrow \mathscr{M}_{k}(\mathbb{C})$ is continuous;

(iv) $f \in \operatorname{DDB}^{k-1}(\Omega)$.

Moreover, if condition (ii) is fulfilled, then $f$ extends to a function $\tilde{f}: \tilde{\Omega} \rightarrow \mathbb{C}$ with $\tilde{\Omega} \supset \Omega$ locally compact such that $\tilde{f}_{\mathrm{op}}: \mathscr{D}_{k}(\tilde{\Omega}) \rightarrow \mathscr{D}_{k}(\mathbb{C})$ is continuous. 
Proof. First assume that $f \in \operatorname{DDB}^{k-1}(\Omega)$. By Proposition 3.2, there exist a locally compact set $\tilde{\Omega}, \Omega \subset \tilde{\Omega} \subset \bar{\Omega}$, and locally Lipschitz functions $F_{j}: \tilde{\Omega}^{[j]} \rightarrow \mathbb{C}(j=$ $1, \ldots, k-1)$ such that

$$
F_{j}\left(z_{1}, \ldots, z_{j}\right)=\Delta\left(z_{1}, \ldots, z_{j}\right) f \quad\left(\left(z_{1}, \ldots, z_{j}\right) \in \Omega^{(j)}\right)
$$

for any $j \in\{1, \ldots, k-1\}$. Define $\tilde{f}: \tilde{\Omega} \rightarrow \mathbb{C}$ as $F_{1}$. It follows from (4-1) that $\tilde{f}$ extends $f$. What is more, since $\tilde{f}$ and $F_{j}$ 's are continuous and $\Omega$ is dense in $\tilde{\Omega}$, we conclude from (4-1) that also

$$
F_{j}\left(z_{1}, \ldots, z_{j}\right)=\Delta\left(z_{1}, \ldots, z_{j}\right) \tilde{f} \quad\left(\left(z_{1}, \ldots, z_{j}\right) \in \tilde{\Omega}^{(j)}\right)
$$

for $j=1, \ldots, k-1$. Since $F_{k-1}$ is locally Lipschitz and symmetric, (4-2) implies that $\tilde{f} \in \operatorname{DDB}^{k-1}(\tilde{\Omega})$. Consequently, thanks to Propositions 3.2 and $3.5, \tilde{f} \in \operatorname{TC}^{k-2}(\tilde{\Omega})$ and

$$
F_{j+1}(z, \ldots, z)=\frac{\tilde{f}^{(j)}(z)}{j !} \quad\left(z \in \tilde{\Omega}^{\prime}, j \in\{0, \ldots, k-2\}\right) .
$$

We will now show that $\tilde{f}_{\text {op }}: \mathscr{M}_{k}^{o}(\tilde{\Omega}) \backslash \mathscr{Z}_{k} \rightarrow \mathscr{M}_{k}(\mathbb{C})$ is continuous (from which one infers (iii)). For simplicity, we put $G:=\mathscr{M}_{k}^{o}(\tilde{\Omega}) \backslash \mathscr{Z}_{k}$. Recall that $\tilde{f}[X]$ makes sense for any $X \in G$ since $X \notin \mathscr{Z}_{k}$ (which means that the algebraic multiplicity of any eigenvalue of $X$ is less than $k$ ) and $\tilde{f}$ is of class TC $^{k-2}$.

Let matrices $X_{1}, X_{2}, \ldots \in G$ converge to $X_{0} \in G$.

First assume that each of $X_{n}$ for $n>0$ is diagonalizable. Write $\operatorname{sp}\left(X_{0}\right)=$ $\left\{\lambda_{0}^{(1)}, \ldots, \lambda_{0}^{(k)}\right\}$. Proposition 4.1 enables us to write (for each $\left.n \geqslant 1\right) \operatorname{sp}\left(X_{n}\right)=$ $\left\{\lambda_{n}^{(1)}, \ldots, \lambda_{n}^{(k)}\right\}$ in a way such that

$$
\lim _{n \rightarrow \infty} \lambda_{n}^{(j)}=\lambda_{0}^{(j)} \quad(j=1, \ldots, k) .
$$

Further, for each $n>0$ write $\mu_{X_{n}}$ in the form $\mu_{X_{n}}(z)=\prod_{j=1}^{k}\left(z-\lambda_{n}^{(j)}\right)^{\nu_{n}(j)}$ where $\nu_{n}(j) \in\{0,1\}$. After passing to a subsequence and rearranging the eigenvalues, we may assume that for some $s \in\{1, \ldots, k\}$ one has $\nu_{n}(j)=1$ for $j \leqslant s$ and $\nu_{n}(j)=0$ for $j>s$ (for any $n>0$ ). Since $\lim _{n \rightarrow \infty} \mu_{X_{n}}\left[X_{n}\right]=\lim _{n \rightarrow \infty} \prod_{j=1}^{s}\left(X_{n}-\lambda_{n}^{(j)} I\right)=$ $\prod_{j=1}^{s}\left(X_{0}-\lambda_{0}^{(j)} I\right)$, we conclude that

$$
\prod_{j=1}^{s}\left(X_{0}-\lambda_{0}^{(j)} I\right)=0
$$

and thus the sets $\operatorname{sp}\left(X_{0}\right)$ and $\left\{\lambda_{0}^{(j)}: j \leqslant s\right\}$ coincide. Further, we infer from the diagonalizability of $X_{n}(n>0)$ and the formula for $\mu_{X_{n}}$ that

$$
\lambda_{n}^{(1)}, \ldots, \lambda_{n}^{(s)} \text { are different for each } n>0 .
$$

So, Lemma 4.2 combined with (4-4) and (4-6) yields

$$
\left(\lambda_{0}^{(1)}, \ldots, \lambda_{0}^{(s)}\right) \in \tilde{\Omega}^{[s]} .
$$

Further, for $n>0$ put

$$
V_{n}(z)=\sum_{q=1}^{s}\left[\Delta\left(\lambda_{n}^{(1)}, \ldots, \lambda_{n}^{(q)}\right) \tilde{f} \cdot \prod_{j<q}\left(z-\lambda_{n}^{(j)}\right)\right] .
$$

It follows from (DD2) that $V_{n}\left(\lambda_{n}^{(j)}\right)=\tilde{f}\left(\lambda_{n}^{(j)}\right)$ for $j \leqslant s$ and hence $\tilde{f}\left[X_{n}\right]=V_{n}\left[X_{n}\right]$. So, we need to show that $\lim _{n \rightarrow \infty} V_{n}\left[X_{n}\right]=\tilde{f}\left[X_{0}\right]$. To this end, we consider three cases. 
First assume that $\operatorname{card}\left(\operatorname{sp}\left(X_{0}\right)\right)=1$. For simplicity, denote by $\lambda$ the unique element of $\operatorname{sp}\left(X_{0}\right)$. Then $\lim _{n \rightarrow \infty} \lambda_{n}^{(j)}=\lambda$ for $j \in\{1, \ldots, s\}$. The fact that $X_{0} \notin \mathscr{Z}_{k}$ combined with (4-7) gives

$$
\left(X_{0}-\lambda I\right)^{\alpha}=0 \quad \text { where } \alpha:=\min (k-1, s)
$$

and therefore

$$
\tilde{f}\left[X_{0}\right]=\sum_{j=0}^{\alpha-1} \frac{\tilde{f}^{(j)}(\lambda)}{j !}\left(X_{0}-\lambda I\right)^{j} .
$$

We obtain from (4-2) and (4-8) that

$$
V_{n}\left[X_{n}\right]=\sum_{q=1}^{\alpha} F_{q}\left(\lambda_{n}^{(1)}, \ldots, \lambda_{n}^{(q)}\right) \cdot \prod_{j<q}\left(X_{n}-\lambda_{n}^{(j)} I\right)
$$

provided $s<k$ and

$V_{n}\left[X_{n}\right]=\sum_{q=1}^{k-1} F_{q}\left(\lambda_{n}^{(1)}, \ldots, \lambda_{n}^{(q)}\right) \cdot \prod_{j<q}\left(X_{n}-\lambda_{n}^{(j)} I\right)+\Delta\left(\lambda_{n}^{(1)}, \ldots, \lambda_{n}^{(k)}\right) \tilde{f} \cdot \prod_{q=1}^{k-1}\left(X_{n}-\lambda_{n}^{(j)} I\right)$

otherwise. Observe that the last summand in the latter formula tends to 0 as $n$ tends to $\infty$, since $\tilde{f} \in \mathrm{DDB}^{k-1}$ and $(X-\lambda I)^{k-1}=0$ (by (4-9)). So, in both the cases we get

$$
\lim _{n \rightarrow \infty} V_{n}\left[X_{n}\right]=\sum_{q=1}^{\alpha} F_{q}(\lambda, \ldots, \lambda) \cdot \prod_{j<q}\left(X_{0}-\lambda I\right)=\sum_{j=0}^{\alpha-1} F_{j+1}(\lambda, \ldots, \lambda)\left(X_{0}-\lambda I\right)^{j} .
$$

So, relations (4-3) and (4-10) finish the proof in case $\operatorname{card}\left(\operatorname{sp}\left(X_{0}\right)\right)=1$.

Now assume that $\operatorname{card}\left(\operatorname{sp}\left(X_{0}\right)\right)>1$. Then $s>1$. Let $\sigma$ be any permutation of $\{1, \ldots, s\}$ such that $\sigma(1) \neq \sigma(s)$. Thanks to (DD1) and (4-2), we may transform (4-8) into

$$
\begin{aligned}
V_{n}(z) & =\sum_{q=1}^{s} \Delta\left(\lambda_{n}^{(\sigma(1))}, \ldots, \lambda_{n}^{(\sigma(q))}\right) \tilde{f} \cdot \prod_{j<q}\left(z-\lambda_{n}^{(\sigma(j))}\right) \\
& =\sum_{q=1}^{s-1} F_{q}\left(\lambda_{n}^{(\sigma(1))}, \ldots, \lambda_{n}^{(\sigma(q))}\right) \cdot \prod_{j<q}\left(z-\lambda_{n}^{(\sigma(j))}\right) \\
& +\frac{F_{s-1}\left(\lambda_{n}^{(\sigma(1))}, \ldots, \lambda_{n}^{(\sigma(s-1))}\right)-F_{s-1}\left(\lambda_{n}^{(\sigma(2))}, \ldots, \lambda_{n}^{(\sigma(s))}\right)}{\lambda_{n}^{(\sigma(1))}-\lambda_{n}^{(\sigma(s))}} \cdot \prod_{j=1}^{s-1}\left(z-\lambda_{n}^{(j)}\right) .
\end{aligned}
$$

Consequently,

$$
\begin{aligned}
& \lim _{n \rightarrow \infty} V_{n}(z)=V(z):=\sum_{q=1}^{s-1} F_{q}\left(\lambda_{0}^{(\sigma(1))}, \ldots, \lambda_{0}^{(\sigma(q))}\right) \cdot \prod_{j<q}\left(z-\lambda_{0}^{(\sigma(j))}\right) \\
& +\frac{F_{s-1}\left(\lambda_{0}^{(\sigma(1))}, \ldots, \lambda_{0}^{(\sigma(s-1))}\right)-F_{s-1}\left(\lambda_{0}^{(\sigma(2))}, \ldots, \lambda_{0}^{(\sigma(s))}\right)}{\lambda_{0}^{(\sigma(1))}-\lambda_{0}^{(\sigma(s))}} \cdot \prod_{j=1}^{s-1}\left(z-\lambda_{0}^{(j)}\right)
\end{aligned}
$$

(the above formula means, in particular, that the formula for $V$ is independent of the permutation $\sigma$ ) and $\lim _{n \rightarrow \infty} V_{n}\left[X_{n}\right]=V\left[X_{0}\right]$. So, the proof will be completed if we show that $V\left[X_{0}\right]=\tilde{f}\left[X_{0}\right]$. To do this, it suffices to check that $V^{(q)}\left(\lambda_{0}^{(j)}\right)=\tilde{f}^{(q)}\left(\lambda_{0}^{(j)}\right)$ for any $j \in\{1, \ldots, s\}$ and $q$ less than the multiplicity of $\lambda_{0}^{(j)}$ as a root of $\mu_{X_{0}}$. To this end, fix $j_{0} \in\{1, \ldots, s\}$ and take any permutation $\tau$ of $\{1, \ldots, s\}$ such that for some $p \in\{1, \ldots, s-1\}, \lambda_{0}^{(\tau(1))}=\ldots=\lambda_{0}^{\tau(p)}=\lambda_{0}^{\left(j_{0}\right)}$ and $\lambda_{0}^{\tau(q)} \neq \lambda_{0}^{\left(j_{0}\right)}$ for $q>p$. (Note that $p<s$ because $\operatorname{card}\left(\operatorname{sp}\left(X_{0}\right)\right)>1$.) For simplicity, put $w:=\lambda_{0}^{\left(j_{0}\right)}$. 
We infer from (4-5) that then the multiplicity of $w$ as a root of $\mu_{X_{0}}$ is not greater than $p$. Thus, we only need to check that $V^{(q)}(w)=\tilde{f}^{(q)}(w)$ for $q=0, \ldots, p-1$. Substituting in (4-11) $\tau$ for $\sigma$, we see that for a suitable polynomial $Q$ one has

$$
V(z)=\sum_{q=0}^{p-1} F_{q+1}(w, \ldots, w)(z-w)^{q}+(z-w)^{p} Q(z) .
$$

The above, combined with (4-3), completes the proof.

Now assume that $X_{n}$ 's (for $n>0$ ) are arbitrary. Fix for a moment $m>0$. Lemma 4.2 implies that there is a sequence $Y_{1}, Y_{2}, \ldots \in \mathscr{D}_{k}(\tilde{\Omega})$ which converges to $X_{m}$. It follows from the first part of the proof that $\lim _{n \rightarrow \infty} \tilde{f}\left[Y_{n}\right]=\tilde{f}\left[X_{m}\right]$ and thus there is $\beta_{m} \in\{1,2,3, \ldots\}$ such that for $X_{m}^{\prime}:=Y_{\beta_{m}}$ one has

$$
\left\|X_{m}^{\prime}-X_{m}\right\| \leqslant \frac{1}{m} \quad \text { and } \quad\left\|\tilde{f}\left[X_{m}^{\prime}\right]-\tilde{f}\left[X_{m}\right]\right\| \leqslant \frac{1}{m} .
$$

The former inequality in (4-12) shows that $\lim _{n \rightarrow \infty} X_{n}^{\prime}=X_{0}$. Since $X_{n}^{\prime} \in \mathscr{D}_{k}(\tilde{\Omega})$, we conclude from the first part of the proof that $\lim _{n \rightarrow \infty} \tilde{f}\left[X_{n}^{\prime}\right]=\tilde{f}\left[X_{0}\right]$ which, combined with the latter inequality in (4-12), yields $\lim _{n \rightarrow \infty} \tilde{f}\left[X_{n}\right]=\tilde{f}\left[X_{0}\right]$. This finishes the proof of implication '(iv) $\Longrightarrow$ (iii)'.

Since implications '(ii) $\Longrightarrow$ (i)' and '(iii) $\Longrightarrow$ (ii)' are trivial, we only need to show that (iv) follows from (i). To this end, fix $\lambda \in \Omega^{\prime}$ and for any $\ell=\left(\lambda_{1}, \ldots, \lambda_{k}\right) \in \Omega^{(k)}$ and a positive real number $\varepsilon$ denote by $A_{\ell, \varepsilon}$ the matrix $\left[a_{p, q}\right]$ such that $a_{j, j}=\lambda_{j}$ $(j=1, \ldots, k), a_{j+1, j}=\varepsilon(j=1, \ldots, k-1)$ and $a_{p, q}=0$ otherwise. Notice that $A_{\ell, \varepsilon} \in \mathscr{D}_{k}(\Omega), \operatorname{sp}\left(A_{\ell, \varepsilon}\right)=\left\{\lambda_{1}, \ldots, \lambda_{k}\right\}$ and $A_{\ell, \varepsilon} \rightarrow \lambda I$ provided $\varepsilon \rightarrow 0$ and $\ell \stackrel{\Omega^{(k)}}{\rightarrow}(\lambda, \ldots, \lambda) \in \mathbb{C}^{k}$. Let $b_{\ell, \varepsilon}$ stand for the bottom left corner of $f\left[A_{\ell, \varepsilon}\right]$. So, if (i) is fulfilled, there are $\delta>0$ and $C \in \mathbb{R}$ such that

$$
\left|b_{\ell, \varepsilon}\right| \leqslant C \quad \text { whenever } \varepsilon<\delta \text { and } \ell \in \prod_{j=1}^{k} B(\lambda, \delta)
$$

(for the definition of $B(\lambda, \delta)$ see (3-2)). Observe that $f\left[A_{\ell, \varepsilon}\right]=V_{\ell, \varepsilon}\left[A_{\ell, \varepsilon}\right]$ where, for $\ell=\left(\lambda_{1}, \ldots, \lambda_{k}\right)$,

$$
V_{\ell, \varepsilon}(z)=\sum_{j=1}^{k} \Delta\left(\lambda_{1}, \ldots, \lambda_{j}\right) f \cdot \prod_{s<j}\left(z-\lambda_{s}\right) .
$$

Write $V_{\ell, \varepsilon}$ in the form $V_{\ell, \varepsilon}(z)=\sum_{j=0}^{k-1} \alpha_{j} z^{j}$ and note that $\alpha_{k-1}=\Delta\left(\lambda_{1}, \ldots, \lambda_{k}\right) f$. Taking this into account, one may check that $b_{\ell, \varepsilon}=\Delta\left(\lambda_{1}, \ldots, \lambda_{k}\right) f \cdot \varepsilon^{k-1}$ (compare with the Opitz formula [10] or [4, Proposition 25]). Now observe that (4-13) holds iff limsup $\sup _{\ell^{\Omega(k)} \rightarrow(\lambda, \ldots, \lambda)}|\Delta(\ell) f|<\infty$, which finishes the proof.

4.4. Remark. One may easily conclude from the above proof that if only $f$ is continuous, then $f_{\text {op }}: \mathscr{D}_{k}(\Omega) \rightarrow \mathscr{D}_{k}(\mathbb{C})$ is continuous at each $X_{0} \in \mathscr{D}_{k}(\Omega)$ with $\operatorname{card}\left(\operatorname{sp}\left(X_{0}\right)\right)=k($ for any $k)$.

Theorem 4.3 shows that whenever $f_{\text {op }}: \mathscr{D}_{k}(\Omega) \rightarrow \mathscr{M}_{k}(\mathbb{C})$ is continuous, it extends to a continuous function of $\mathscr{M}_{k}^{o}(\Omega) \backslash \mathscr{Z}_{k}$ into $\mathscr{M}_{k}(\mathbb{C})$. Taking this into account, it seems to be interesting the question of when $f_{\text {op }}$ extends to a continuous function of $\mathscr{M}^{\circ}(\Omega)$. A full answer to this problem gives

4.5. Proposition. For a function $f: \Omega \rightarrow \mathbb{C}$ and arbitrary $k \geqslant 2$ the following conditions are equivalent:

(i) $f_{\text {op }}: \mathscr{D}_{k}(\Omega) \rightarrow \mathscr{M}_{k}(\mathbb{C})$ extends to a continuous function of $\mathscr{M}_{k}^{o}(\Omega)$;

(ii) $f$ is of class $\mathrm{TC}^{k-1}$ and $f_{\text {op }}: \mathscr{M}_{k}^{o}(\Omega) \rightarrow \mathscr{M}_{k}(\mathbb{C})$ is continuous; 
(iii) $f \in \operatorname{DDC}^{k-1}(\Omega)$.

Proof. As in the previous proof, first we assume that $f \in \operatorname{DDC}^{k-1}(\Omega)$. Our aim is to show (ii). We know from Proposition 3.5 and Theorem 4.3 that $f \in \operatorname{TC}^{k-1}(\Omega)$. Fix arbitrary $X_{0} \in \mathscr{M}_{k}^{o}(\Omega)$ and let matrices $X_{1}, X_{2}, \ldots \in \mathscr{M}_{k}^{o}(\Omega)$ converge to $X_{0}$. The argument presented in the last part of the proof of implication '(iv) $\Longrightarrow$ (iii)' in Theorem 4.3 ensures us that we may assume each of $X_{n}(n>0)$ is diagonalizable. Then if $X_{0} \notin \mathscr{Z}_{k}$, we deduce from Theorem 4.3 that $\lim _{n \rightarrow \infty} f\left[X_{n}\right]=f\left[X_{0}\right]$. Hence we may and do assume that $X_{0} \in \mathscr{Z}_{k}$. Let $F_{j}: \Omega^{[j]} \rightarrow \mathbb{C}(j=1, \ldots, n)$ be as in Proposition 3.3. Denote by $\lambda$ the unique element of $\operatorname{sp}\left(X_{0}\right)$. Write $\operatorname{sp}\left(X_{n}\right)=$ $\left\{\lambda_{n}^{(1)}, \ldots, \lambda_{n}^{(k)}\right\}(n>0)$. Then $\lim _{n \rightarrow \infty} \lambda_{n}^{(j)}=\lambda(j=1, \ldots, k)$. Mimicing the proof of Theorem 4.3, we may assume that for some $s \in\{1, \ldots, k\}, \mu_{X_{n}}(z)=$ $\prod_{j=1}^{s}\left(z-\lambda_{n}^{(j)}\right)$ for any $n>0$. Observe that $\lim _{n \rightarrow \infty} \mu_{X_{n}}\left[X_{n}\right]=\left(X_{0}-\lambda I\right)^{s}$, from which we infer that $s=k$ (since $\left.X_{0} \in \mathscr{Z}_{k}\right)$. This implies that $\operatorname{card}\left(\operatorname{sp}\left(X_{n}\right)\right)=k$ for positive $n$ and thus:

$$
\begin{aligned}
f\left[X_{n}\right]=\sum_{q=1}^{k} \Delta\left(\lambda_{n}^{(1)}, \ldots, \lambda_{n}^{(q)}\right) f \cdot \prod_{j<q}\left(X_{n}-\lambda^{(j)} I\right) & \\
& =\sum_{q=1}^{k} F_{q}\left(\lambda_{n}^{(1)}, \ldots, \lambda_{n}^{(q)}\right) \cdot \prod_{j<q}\left(X_{n}-\lambda^{(j)} I\right) .
\end{aligned}
$$

Consequently, $\lim _{n \rightarrow \infty} f\left[X_{n}\right]=\sum_{q=1}^{k} F_{q}(\lambda, \ldots, \lambda) \cdot\left(X_{0}-\lambda I\right)^{q-1}$. Now an application of Proposition 3.5 allows us to transform the last equality into $\lim _{n \rightarrow \infty} f\left[X_{n}\right]=$ $\sum_{q=1}^{k} \frac{f^{(q-1)}(\lambda)}{(q-1) !}\left(X_{0}-\lambda I\right)^{q-1}=f\left[X_{0}\right]$ and we are done.

Since (i) is readily implied by (ii), it remains to show that (iii) follows from (i). To this end, fix $\lambda \in \Omega^{\prime}$. Denote by $A$ the matrix $\left[a_{p, q}\right]$ such that $a_{p, q}=1$ when $p=q+1$ and $a_{p, q}=0$ otherwise. For $\ell \in \Omega^{(k)}$ and $\varepsilon>0$ let $A_{\ell, \varepsilon}$ and $b_{\ell, \varepsilon}$ be as in the proof of Theorem 4.3. Note that $A+\lambda I \in \mathscr{M}_{k}^{o}(\Omega), A_{\ell, \varepsilon} \in \mathscr{D}_{k}(\Omega)$ and $A_{\ell, 1+\varepsilon}$ tends to $A+\lambda I$ as

$$
\varepsilon \rightarrow 0 \quad \text { and } \quad \ell \stackrel{\Omega^{(k)}}{\rightarrow}(\lambda, \ldots, \lambda) \in \mathbb{C}^{k} .
$$

We deduce from (i) that $f\left[A_{\ell, 1+\varepsilon}\right]$ converges when (4-14) holds. Consequently, $b_{\ell, 1+\varepsilon}$ converges as well. But $b_{\ell, 1+\varepsilon}=\Delta(\ell) f \cdot(1+\varepsilon)^{k-1}$ and therefore $\Delta(\ell) f$ has a finite limit as $\ell \stackrel{\Omega^{(k)}}{\rightarrow}(\lambda, \ldots, \lambda)$, which finishes the proof.

Now we are ready to give

Proof of Theorem 1.2. Let us start with (A). It follows from Theorem 4.3 and Proposition 4.5 that each of the conditions (i) and (ii) (in point (A)) implies that $f \in \operatorname{DDC}^{1}(\Omega)$ (recall that $k \geqslant 3$ and take into account Proposition 3.2). So, we infer from Proposition 3.5 that $f \in \mathrm{TC}^{1}(\Omega)$ and consequently $f$ is holomorphic, since $\Omega$ is open. Conversely, if $f$ is holomorphic, then $f_{\text {op }}: \mathscr{M}_{j}(\Omega) \rightarrow \mathscr{M}_{j}(\mathbb{C})$ is holomorphic for any $j$ as well, which is readily followed by (i) and (ii).

We pass to point (B). It follows from Theorem 4.3 each of the conditions (i) and (ii) is equivalent to the fact that $f \in \operatorname{DDB}^{k-1}(\Omega)$ (notice that here $\mathscr{M}_{k}(\Omega)=$ $\mathscr{M}_{k}^{o}(\Omega)$ ). But in these settings this last property is equivalent to (iii), thanks to Corollary 3.6. Finally, $f_{\text {op }}: \mathscr{D}_{k}(\Omega) \rightarrow \mathscr{M}_{k}(\mathbb{C})$ extends to a continuous function of $\mathscr{M}_{k}(\Omega)$ iff $f \in \operatorname{DDC}^{k-1}(\Omega)$ (by Proposition 4.5 ) or, equivalently, iff $f \in C^{k-1}(\Omega)$ (see Proposition 2.2 and Theorem 3.4 and note that being of class DDC ${ }^{k-1}$ is a local property; cf. the proofs of Proposition 3.5 and Corollary 3.6). 
4.6. Example. Let us show that being of class $\mathrm{TC}^{\infty}$ is insufficient for the continuity of the extended functional calculus. Let $\Omega=\{0\} \cup\{1 / n: n \geqslant 2\} \cup\left\{1 / n+3^{-n}: n \geqslant\right.$ 2 \} and let $f: \Omega \rightarrow \mathbb{C}$ be defined as follows: $f(0)=f(1 / n)=0$ and $f\left(1 / n+3^{-n}\right)=$ $2^{-n}$ for each $n>0$. Notice that $\Omega$ is compact and $\lim _{x \rightarrow 0} f(x) / x^{k}=0$ for any $k$. This yields that $f \in \mathrm{TC}^{\infty}(\Omega)$ (indeed, $\Omega^{\prime}=\{0\}$ and $f^{(k)}(0)=0$ for each $k$ ). However, $f \notin \operatorname{DDB}^{1}(\Omega)$ because $\Delta\left(1 / n+3^{-n}, 1 / n\right) f=(3 / 2)^{n}$. So, $f_{\text {op }}: \mathscr{D}_{k}(\Omega) \rightarrow$ $\mathscr{M}_{k}(\mathbb{C})$ is discontinuous for any $k>1$ (by Theorem 4.3 ).

The above example shows also that the geometric shape of the set $\Omega$ matters when compering different concepts of differentiability.

\section{Aspects of Uniform CONTINUity}

When dealing with functional calculus for matrices, the adjective 'uniform' may refer to two different aspects of uniformity, namely:

- 'uniform continuity' of $f_{\text {op }}: \mathscr{D}_{k}(\Omega) \rightarrow \mathscr{M}_{k}(\mathbb{C})$ as independent of the point at which the continuity is investigated; that is: for any $\varepsilon>0$ there is $\delta>0$ such that $\|f[X]-f[Y]\| \leqslant \varepsilon$ whenever $X, Y \in \mathscr{D}_{k}(\Omega)$ are such that $\|X-Y\| \leqslant \delta$ (here $k$ is fixed);

- 'uniform continuity' of $f_{\text {op }}: \mathscr{D}_{k}(\Omega) \rightarrow \mathscr{M}_{k}(\mathbb{C})$ as independent of $k$; for example: for any $\lambda \in \Omega^{\prime}$ and each $\varepsilon>0$ there is $\delta>0$ such that $\|f[X]-f[\lambda I]\| \leqslant \varepsilon$ provided $X \in \mathscr{D}_{n}(\Omega)$ is such that $\|X-\lambda I\| \leqslant \delta$ (here $n \geqslant 1$ is arbitrary).

In this section we discuss both the above approaches. We begin with a useful

5.1. Proposition. For two functions $f: \Omega \rightarrow \mathbb{C}$ and $F: \mathscr{M}_{k}^{o}(\tilde{\Omega}) \rightarrow \mathscr{M}_{k}(\mathbb{C})$ where $\Omega \subset \tilde{\Omega} \subset \bar{\Omega}$ the following conditions are equivalent:

(i) $F$ is continuous and $F(X)=f[X]$ for $X \in \mathscr{D}_{k}(\Omega)$;

(ii) $f$ extends to a function $\tilde{f}: \tilde{\Omega} \rightarrow \mathbb{C}$ of class $\operatorname{DDC}^{k-1}$ such that $F(X)=\tilde{f}[X]$ for any $X \in \mathscr{D}_{k}(\Omega)$.

Proof. We conclude from Proposition 4.5 that (i) follows from (ii). Now assume (i) holds and observe that for any $\lambda \in \Omega, F(\lambda I)=f(\lambda) I$. So, it follows from the continuity of $F$ and the density of $\Omega$ in $\tilde{\Omega}$ that for and $\lambda \in \tilde{\Omega}, F(\lambda I)$ is a scalar multiple of the identity matrix $I$. This notice enables us to define a function $\tilde{f}: \tilde{\Omega} \rightarrow \mathbb{C}$ by the formula $F(\lambda I)=\tilde{f}(\lambda) I$. It is clear that $\tilde{f}$ extends $f$ and is continuous. Fix $X \in \mathscr{D}_{k}(\Omega)$ and take an invertible $k \times k$ matrix $P$ such that $D:=P X P^{-1}$ is diagonal. It is easily seen that there is a sequence $D_{1}, D_{2}, \ldots$ of diagonal matrices belonging to $\mathscr{D}_{k}(\Omega)$ which converges to $D$. It follows from the continuity of $\tilde{f}$ that $\lim _{n \rightarrow \infty} \tilde{f}\left[D_{n}\right]=\tilde{f}[D]$ and thus

$$
\begin{aligned}
& \tilde{f}[X]=P^{-1} \tilde{f}[D] P=\lim _{n \rightarrow \infty}\left(P^{-1} f\left[D_{n}\right] P\right)=\lim _{n \rightarrow \infty} f\left[P^{-1} D_{n} P\right] \\
&=F\left(P^{-1} D P\right)=F(X) .
\end{aligned}
$$

So, we see that $\tilde{f}_{\text {op }}: \mathscr{D}_{k}(\tilde{\Omega}) \rightarrow \mathscr{M}_{k}(\mathbb{C})$ extends to a continuous function of $\mathscr{M}_{k}^{o}(\tilde{\Omega})$ into $\mathscr{M}_{k}(\mathbb{C})$ (namely, $F$ ). Consequently, $\tilde{f} \in \operatorname{DDC}^{k-1}(\tilde{\Omega})$, by Proposition 4.5 . Then also $\tilde{f}_{\text {op }}: \mathscr{M}_{k}^{o}(\tilde{\Omega}) \rightarrow \mathscr{M}_{k}(\mathbb{C})$ is continuous and therefore $F(X)=\tilde{f}[X]$ for any $X \in \mathscr{M}_{k}^{o}(\tilde{\Omega})$, since these two functions coincide on a dense set (cf. Lemma 4.2).

First we shall characterize those functions $f: \Omega \rightarrow \mathbb{C}$ for which $f_{\text {op }}: \mathscr{D}_{2}(\Omega) \rightarrow$ $\mathscr{M}_{2}(\Omega)$. As the following result shows, this characterization (in general) is somewhat strange.

5.2. Lemma. For a function $f: \Omega \rightarrow \mathbb{C}$ the following conditions are equivalent:

(i) $f_{\text {op }}: \mathscr{D}_{2}(\Omega) \rightarrow \mathscr{M}_{2}(\mathbb{C})$ is uniformly continuous; 
(ii) $f$ is Lipschitz, the formula

$$
z+w \mapsto f(z)+f(w) \quad(z, w) \in \Omega^{(2)}
$$

well defines a uniformly continuous function on $\left\{z+w:(z, w) \in \Omega^{(2)}\right\}$, and there exists $\delta>0$ such that

(5-2) $\Delta(z, w) f=\Delta\left(z^{\prime}, w^{\prime}\right) f \quad$ if $(z, w),\left(z^{\prime}, w^{\prime}\right) \in \Omega^{(2)}$ and $\left|(z+w)-\left(z^{\prime}+w^{\prime}\right)\right| \leqslant \delta$.

Proof. First assume $f_{\text {op }}: \mathscr{D}_{2}(\Omega) \rightarrow \mathscr{M}_{2}(\mathbb{C})$ is uniformly continuous. We want to prove that all conditions of (ii) are fulfilled. To show that $f$ is Lipschitz, we need to check that the function $(z, w) \ni \Omega^{(2)} \mapsto \Delta(z, w) f \in \mathbb{C}$ is bounded. To this end, fix a sequence $\left(z_{1}, w_{1}\right),\left(z_{2}, w_{2}\right), \ldots$ of arbitrary elements of $\Omega^{(2)}$ and consider the matrices $A_{n}=\left(\begin{array}{cc}z_{n} & 0 \\ 0 & w_{n}\end{array}\right)$ and $A_{n}^{\prime}=\left(\begin{array}{cc}z_{n} & 0 \\ \varepsilon_{n} & w_{n}\end{array}\right)$ where $\mathscr{E}=\left(\varepsilon_{1}, \varepsilon_{2}, \ldots\right)$ is a sequence convergent to 0 . Then $A_{n}, A_{n}^{\prime} \in \mathscr{D}_{2}(\Omega)$ and $\lim _{n \rightarrow \infty}\left\|A_{n}-A_{n}^{\prime}\right\|=0$. So, we conclude from (i) that the sequence $\gamma(\mathscr{E})=\left(\gamma_{1}(\mathscr{E}), \gamma_{2}(\mathscr{E}), \ldots\right)$ of bottom left corners of $f\left[A_{n}\right]-f\left[A_{n}^{\prime}\right]$ is bounded. But $\gamma_{n}(\mathscr{E})=\Delta\left(z_{n}, w_{n}\right) \varepsilon_{n}$. Since this sequence is bounded for any $\mathscr{E}$ convergent to 0 , we conclude that $\sup _{n \geqslant 1}\left|\Delta\left(z_{n}, w_{n}\right) f\right|<\infty$.

Now we claim that there is $\delta>0$ such that (5-2) is satisfied. For if not, there are two sequences $\left(z_{1}, w_{1}\right),\left(z_{2}, w_{2}\right), \ldots$ and $\left(z_{1}^{\prime}, w_{1}^{\prime}\right),\left(z_{2}^{\prime}, w_{2}^{\prime}\right), \ldots$ of elements of $\Omega^{(2)}$ such that

$$
\varepsilon_{n}:=\left(z_{n}^{\prime}+w_{n}^{\prime}\right)-\left(z_{n}+w_{n}\right) \rightarrow 0 \quad(n \rightarrow \infty)
$$

and $\kappa_{n}:=\Delta\left(z_{n}, w_{n}\right) f-\Delta\left(z_{n}^{\prime}, w_{n}^{\prime}\right) f$ is nonzero for each $n$. Fix a sequence $a_{1}, a_{2}, \ldots$ of nonzero complex numbers, put $b_{n}:=\frac{z_{n}\left(w_{n}+\varepsilon_{n}\right)-z_{n}^{\prime} w_{n}^{\prime}}{a_{n}}$ and define matrices $A_{n}$ and $A_{n}^{\prime}(n>0)$ as follows: $A_{n}=\left(\begin{array}{cc}z_{n} & 0 \\ a_{n} & w_{n}\end{array}\right), A_{n}^{\prime}=\left(\begin{array}{cc}z_{n} & b_{n} \\ a_{n} & w_{n}+\varepsilon_{n}\end{array}\right)$. It is clear that $A_{n} \in \mathscr{D}_{2}(\Omega)$ and $\operatorname{sp}\left(A_{n}\right)=\left\{z_{n}, w_{n}\right\}$. Observe that $\operatorname{tr}\left(A_{n}^{\prime}\right)=z_{n}^{\prime}+w_{n}^{\prime}$, by $(5-3)$ (' $\operatorname{tr}(X)$ ' is the trace of a matrix $X)$, and $\operatorname{det}\left(A_{n}^{\prime}\right)=z_{n}^{\prime} w_{n}^{\prime}$, by the definition of $b_{n}$. We conclude that $\operatorname{sp}\left(A_{n}^{\prime}\right)=\left\{z_{n}^{\prime}, w_{n}^{\prime}\right\}$ and thus $A_{n}^{\prime} \in \mathscr{D}_{2}(\Omega)\left(\right.$ since $\left.z_{n}^{\prime} \neq w_{n}^{\prime}\right)$. Notice that $\lim _{n \rightarrow \infty}\left\|A_{n}-A_{n}^{\prime}\right\|=0$ iff

$$
\lim _{n \rightarrow \infty} b_{n}=0
$$

(thanks to (5-3)). Further, it follows from the definition of the extended functional calculus that $f\left[A_{n}\right]=f\left(z_{n}\right) I+\Delta\left(z_{n}, w_{n}\right) f \cdot\left(A_{n}-z_{n} I\right)$ and $f\left[A_{n}^{\prime}\right]=f\left(z_{n}^{\prime}\right) I+$ $\Delta\left(z_{n}^{\prime}, w_{n}^{\prime}\right) f \cdot\left(A_{n}^{\prime}-z_{n}^{\prime} I\right)$ (where $I$ is the $2 \times 2$ unit matrix). Consequently, if $\gamma_{n}$ denotes the bottom left corner of $f\left[A_{n}\right]-f\left[A_{n}^{\prime}\right]$, then $\gamma_{n}=\kappa_{n} a_{n}$. Since $\kappa_{n} \neq 0$, we see that it is possible to find $a_{n}$ 's such that (5-4) holds and $\lim _{n \rightarrow \infty}\left|\gamma_{n}\right|=\infty$. But then, for such $a_{n}$ 's, $\lim _{n \rightarrow \infty}\left\|A_{n}-A_{n}^{\prime}\right\|=0$ and the sequence of $f\left[A_{n}\right]-f\left[A_{n}^{\prime}\right]$ $(n>0)$ is unbounded, which contradicts (i). This finishes the proof of the last claim in (ii). Additionally, observe that if we continue the above argument, i.e. starting from (5-3), then choosing $a_{n}$ 's in a way such that (5-4) holds, and defining $A_{n}$ and $A_{n}^{\prime}$ as above, we will infer from (i) that $\lim _{n \rightarrow \infty}\left\|f\left[A_{n}\right]-f\left[A_{n}^{\prime}\right]\right\|=0$. Consequently, $\lim _{n \rightarrow \infty}\left(\operatorname{tr}\left(f\left[A_{n}\right]\right)-\operatorname{tr}\left(f\left[A_{n}^{\prime}\right]\right)\right)=0$. But $\operatorname{tr}\left(f\left[A_{n}\right]\right)=f\left(z_{n}\right)+f\left(w_{n}\right)$ (since $\left.\operatorname{sp}\left(f\left[A_{n}\right]\right)=\left\{z_{n}, w_{n}\right\}\right)$ and analogously $\operatorname{tr}\left(f\left[A_{n}^{\prime}\right]\right)=f\left(z_{n}^{\prime}\right)+f\left(w_{n}^{\prime}\right)$. This shows that if $\left(z_{n}, w_{n}\right),\left(z_{n}^{\prime}, w_{n}^{\prime}\right) \in \Omega^{(2)}$ and $\lim _{n \rightarrow \infty}\left|\left(z_{n}+w_{n}\right)-\left(z_{n}^{\prime}, w_{n}^{\prime}\right)\right|=0$, then $\lim _{n \rightarrow \infty}\left|\left(f\left(z_{n}\right)+f\left(w_{n}\right)\right)-\left(f\left(z_{n}^{\prime}\right)+f\left(w_{n}^{\prime}\right)\right)\right|=0$ as well. Equivalently, (5-1) well defines a uniformly continuous function and the proof of (ii) is complete.

Now assume that all conditions of (ii) are fulfilled. Fix two sequences $A_{1}, A_{2}, \ldots$ and $A_{1}^{\prime}, A_{2}^{\prime}, \ldots$ of members of $\mathscr{D}_{2}(\Omega)$ such that $\lim _{n \rightarrow \infty}\left\|A_{n}-A_{n}^{\prime}\right\|=0$. Our aim is to show that $\lim _{n \rightarrow \infty}\left\|f\left[A_{n}\right]-f\left[A_{n}^{\prime}\right]\right\|=0$ as well. To this end, we consider three cases. 
If both $A_{n}$ and $A_{n}^{\prime}$ are scalar multiples of the unit matrix, then clearly $\| f\left[A_{n}\right]-$ $f\left[A_{n}^{\prime}\right]\|\leqslant L(f) \cdot\| A_{n}-A_{n}^{\prime} \|$ where $L(f)$ is a Lipschitz constant for $f$. In that case the assertion is therefore immediate.

If, for example, $A_{n}^{\prime}$ is a scalar multiple of the unit matrix, say $A_{n}^{\prime}=\gamma_{n} I$, and $A_{n}$ is not, then write $\operatorname{sp}\left(A_{n}\right)=\left\{z_{n}, w_{n}\right\}$ and note that $\left(z_{n}, w_{n}\right) \in \Omega^{(2)}$ and $\operatorname{sp}\left(A_{n}-A_{n}^{\prime}\right)=\left\{z_{n}-\gamma_{n}, w_{n}-\gamma_{n}\right\}$. Consequently, $\lim _{n \rightarrow \infty}\left|z_{n}-\gamma_{n}\right|=0$, and $f\left[A_{n}\right]=f\left(z_{n}\right) I+\Delta\left(z_{n}, w_{n}\right) f \cdot\left(A_{n}-z_{n} I\right)$ and $f\left[A_{n}^{\prime}\right]=f\left(\gamma_{n}\right) I$. So,

$$
\begin{array}{r}
\left\|f\left[A_{n}\right]-f\left[A_{n}^{\prime}\right]\right\|=\left\|\left(f\left(z_{n}\right)-f\left(\gamma_{n}\right)\right) I+\Delta\left(z_{n}, w_{n}\right) f \cdot\left[\left(A_{n}-A_{n}^{\prime}\right)+\left(\gamma_{n}-z_{n}\right) I\right]\right\| \\
\leqslant 2 L(f)\left|z_{n}-\gamma_{n}\right|+L(f)\left\|A_{n}-A_{n}^{\prime}\right\| \rightarrow 0 \quad(n \rightarrow \infty) .
\end{array}
$$

Finally, we assume that neither $A_{n}$ nor $A_{n}^{\prime}$ is not a scalar multiple of the unit matrix. Then $\operatorname{sp}\left(A_{n}\right)=\left\{z_{n}, w_{n}\right\}$ and $\operatorname{sp}\left(A_{n}^{\prime}\right)=\left(z_{n}^{\prime}, w_{n}^{\prime}\right)$ for some $\left(z_{n}, w_{n}\right),\left(z_{n}^{\prime}, w_{n}^{\prime}\right) \in$ $\Omega^{(2)}$. For simplicity, denote by $F$ the function defined by $(5-1)$. So, $F(z+w)=$ $f(z)+f(w)$ for any $(z, w) \in \Omega^{(2)}$. Consequently, $F\left(\operatorname{tr}\left(A_{n}\right)\right)=\operatorname{tr}\left(f\left[A_{n}\right]\right)$ and $F\left(\operatorname{tr}\left[A_{n}^{\prime}\right]\right)=\operatorname{tr}\left(f\left[A_{n}^{\prime}\right]\right)$. We conclude from the uniform continuity of $F$ that

$$
\lim _{n \rightarrow \infty}\left|\operatorname{tr}\left(f\left[A_{n}\right]\right)-\operatorname{tr}\left(f\left[A_{n}^{\prime}\right]\right)\right|=0
$$

(because $\left.\left|\operatorname{tr}\left(A_{n}\right)-\operatorname{tr}\left(A_{n}^{\prime}\right)\right| \rightarrow 0\right)$. Further, it follows from (5-2) that $\Delta\left(z_{n}, w_{n}\right) f=$ $\Delta\left(z_{n}^{\prime}, w_{n}^{\prime}\right) f$ for almost all $n$. Hence, we may assume that for all $n, \Delta\left(z_{n}, w_{n}\right) f=$ $\Delta\left(z_{n}^{\prime}, w_{n}^{\prime}\right) f=: \varrho_{n}$. Recall that $\left|\varrho_{n}\right| \leqslant L(f)$. A straightforward calculation shows that $f\left(z_{n}\right)-\Delta\left(z_{n}, w_{n}\right) f \cdot z_{n}=\frac{f\left(z_{n}\right)+f\left(w_{n}\right)}{2}-\Delta\left(z_{n}, w_{n}\right) f \cdot \frac{z_{n}+w_{n}}{2}$ (and similarly for $\left.\left(z_{n}^{\prime}, w_{n}^{\prime}\right)\right)$. So, $f\left[A_{n}\right]=f\left(z_{n}\right) I+\Delta\left(z_{n}, w_{n}\right) f \cdot\left(A_{n}-z_{n} I\right)=\frac{1}{2}\left(\operatorname{tr}\left(f\left[A_{n}\right]\right)-\right.$ $\left.\varrho_{n} \operatorname{tr}\left(A_{n}\right)\right) I+\varrho_{n} A_{n}$ and a similar formula for $f\left[A_{n}^{\prime}\right]$. Finally, taking into account (5-5), we obtain

$$
\begin{gathered}
\left\|f\left[A_{n}\right]-f\left[A_{n}^{\prime}\right]\right\| \leqslant \frac{1}{2}\left|\operatorname{tr}\left(f\left[A_{n}\right]\right)-\operatorname{tr}\left(f\left[A_{n}^{\prime}\right]\right)\right|+\left|\varrho_{n}\right| \cdot\left(\frac{\left|\operatorname{tr}\left(A_{n}-A_{n}^{\prime}\right)\right|}{2}+\left\|A_{n}-A_{n}^{\prime}\right\|\right) \\
\leqslant \frac{1}{2}\left|\operatorname{tr}\left(f\left[A_{n}\right]\right)-\operatorname{tr}\left(f\left[A_{n}^{\prime}\right]\right)\right|+2 L(f)\left\|A_{n}-A_{n}^{\prime}\right\| \rightarrow 0 \quad(n \rightarrow \infty)
\end{gathered}
$$

which finishes the proof.

For simplicity, let us call a function $f: \Omega \rightarrow \mathbb{C}$ affine (resp. affine on a set $A \subset \Omega$ ) iff there exist $a, b \in \mathbb{C}$ such that $f(z)=a z+b$ for any $z \in \Omega$ (resp. for any $z \in A)$.

As consequences of Lemma 5.2, we obtain the next three results.

5.3. Proposition. Let $\Omega$ be a subset of $\mathbb{C}$ such that $\operatorname{card}(\Omega)>1$ and

$$
\inf \left\{|z-w|:(z, w) \in \Omega^{(2)}\right\}=0 .
$$

Then for any function $f: \Omega \rightarrow \mathbb{C}$ the following conditions are equivalent:

(i) $f_{\text {op }}: \mathscr{D}_{2}(\Omega) \rightarrow \mathscr{M}_{2}(\mathbb{C})$ is uniformly continuous;

(ii) there exist positive real numbers $\varepsilon$ and $M$ such that $\|f[X]-f[Y]\| \leqslant M$ whenever $X, Y \in \mathscr{D}_{2}(\Omega)$ are such that $\|X-Y\| \leqslant \varepsilon$;

(iii) $f$ is affine.

Proof. We only need to prove that (iii) follows from (ii). Notice that the proof of Lemma 5.2 shows that if (ii) is fulfilled, then there is $\delta>0$ such that (5-2) holds (see Lemma 5.2). Then it follows from (5-6) that there are $z_{0}, z_{1} \in \Omega$ with $0<\left|z_{0}-z_{1}\right|<\delta$. Observe that (5-2) implies that

$$
\Delta\left(z_{0}, z\right) f=\Delta\left(z_{1}, z\right) f \quad \text { for any } z \in \Omega \backslash\left\{z_{0}, z_{1}\right\} .
$$

It is an elementary observation that (5-7) is equivalent to

$$
f(z)=\Delta\left(z_{0}, z_{1}\right) f \cdot z+\frac{f\left(z_{0}\right) z_{1}-f\left(z_{1}\right) z_{0}}{z_{1}-z_{0}} \quad(z \in \Omega)
$$


which finishes the proof.

5.4. Corollary. If $\Omega$ is a subset of $\mathbb{C}$ such that for some $\varepsilon>0,\left|(z+w)-\left(z^{\prime}+w^{\prime}\right)\right| \geqslant \varepsilon$ for any two distinct pairs $(z, w),\left(z^{\prime}, w^{\prime}\right) \in \Omega^{(2)}$, then for an arbitrary function $f: \Omega \rightarrow \mathbb{C}, f_{\text {op }}: \mathscr{D}_{2}(\Omega) \rightarrow \mathscr{M}_{2}(\mathbb{C})$ is uniformly continuous iff $f$ is Lipschitz.

The proof of Corollary 5.4 is based on Lemma 5.2 and we leave it to the reader.

5.5. Corollary. Let $\Omega$ be a subset of $\mathbb{C}$ which is symmetric with respect to some $\alpha \in \mathbb{C}$; that is, $2 \alpha-z \in \Omega$ for any $z \in \Omega$. If $f_{\text {op }}: \mathscr{D}_{2}(\Omega) \rightarrow \mathscr{M}_{2}(\Omega)$ is uniformly continuous (where $f: \Omega \rightarrow \mathbb{C}$ ), then $f$ is affine on $\Omega \backslash\{\alpha\}$. In particular, if $\Omega$ is an additive subgroup of $\mathbb{C}$, then $f_{\mathrm{op}}: \mathscr{D}_{2}(\Omega) \rightarrow \mathscr{M}_{2}(\mathbb{C})$ is uniformly continuous iff $f$ is affine.

Proof. Assume $f_{\text {op }}: \mathscr{D}_{2}(\Omega) \rightarrow \mathscr{M}_{2}(\mathbb{C})$ is uniformly continuous. Fix $w \in \Omega \backslash\{\alpha\}$ and put $m=\Delta(w, 2 \alpha-w) f$. Note that for any $z \in \Omega \backslash\{\alpha\}$ we have $(z, 2 \alpha-z),(w, 2 \alpha-$ $w) \in \Omega^{(2)}$ and $z+(2 \alpha-z)=w+(2 \alpha-w)$. Consequently, $\Delta(z, 2 \alpha-z) f=m$, thanks to (5-2), and $f(z)+f(2 \alpha-z)=f(w)+f(2 \alpha-w)$, by (5-1) (see Lemma 5.2). We infer from these two equalities that $f(z)=m(z-\alpha)+\frac{f(w)+f(2 \alpha-w)}{2}$ and hence $f$ is affine on $\Omega \backslash\{\alpha\}$.

Now if $\Omega$ is a nontrivial additive group, then $0 \in \Omega^{\prime}$ or there is $x \in \Omega$ such that $\frac{1}{2} x \notin \Omega$. In the first case the assertion follows from Proposition 5.3, while in the second from the above prove (since $\Omega$, being a group, is symmetric with respect to $\frac{1}{2} x$ and $\Omega \backslash\left\{\frac{1}{2} x\right\}=\Omega$ ).

5.6. Example. As the following somewhat strange example shows, the assertion of Corollary 5.5 cannot be strengthened in general. Let $\Omega=\left\{k^{3}: k \in \mathbb{Z}\right\}$ and $f: \Omega \rightarrow \mathbb{C}$ be arbitrary. We claim that $f_{\text {op }}: \Omega \rightarrow \mathbb{C}$ is uniformly continuous iff $f$ is affine on $\Omega \backslash\{0\}$ (so, $f(0)$ may be chosen independently of other values of $f$ ). The necessity of the latter condition follows from Corollary 5.5. To see its sufficiency, we involve Lemma 5.2. It is easily seen that if $f$ is affine on $\Omega \backslash\{0\}$, then automatically $f$ is Lipschitz (on $\Omega$ ). So, taking into account Lemma 5.2 , it is enough to check that if $(k, l),(m, 0) \in \Omega^{(2)}$ and $k+l=m+0$, then either $k$ or $l$ is zero. But this simply follows from the last Fermat theorem (for exponent 3 ).

The above results show that the characterization of those functions $f: \Omega \rightarrow \mathbb{C}$ for which $f_{\text {op }}: \mathscr{D}_{k}(\Omega) \rightarrow \mathscr{M}_{k}(\mathbb{C})$ is uniformly continuous for $k=2$ does depend on the geometry of $\Omega$. The situation changes when $k \geqslant 3$, as shown by Proposition 1.4, the proof of which we now turn to.

Proof of Proposition 1.4. Since the case when $k=2$ and $\bar{\Omega}^{\prime} \neq \varnothing$ follows from Proposition 5.3, we may and do assume that $k \geqslant 3$. We only need to explain why (iii) follows from (i). First of all observe that $f$ is affine iff $\Delta(x, y, z) f=0$ for any $(x, y, z) \in \Omega^{(3)}$. For a fixed $(x, y, z) \in \Omega^{(3)}$ denote by $A(w)$ (where $w \in \mathbb{C}$ ) the $k \times k$ matrix $\left[a_{p, q}\right]$ such that $a_{1,1}=x, a_{2,2}=y, a_{p, p}=z$ for $p>2, a_{2,1}=a_{3,2}=w$ and $a_{p, q}=0$ otherwise. It is easy to check that $A(w) \in \mathscr{D}_{2}(\Omega), \operatorname{sp}(A(w))=\{x, y, z\}$ and the function $\mathbb{C} \ni x \mapsto A(w) \in \mathscr{M}_{k}(\mathbb{C})$ is uniformly continuous. Consequently, if (i) is satisfied and $b(w)$ denotes the entry of $f[A(w)]$ which lies in its third row and first column, then there exists $\delta>0$ such that $\left|b(w)-b\left(w^{\prime}\right)\right| \leqslant M$ whenever $\left|w-w^{\prime}\right| \leqslant \delta$ (where $M$ is as in (i)). But $b(w)=\Delta(x, y, z) f \cdot w^{2}$ and hence the function $\mathbb{R} \ni w \mapsto|b(w)-b(w+\delta)| \in \mathbb{R}$ is unbounded unless $\Delta(x, y, z)=0$. This finishes the proof.

Proposition 1.4 says that the problem of characterizing 'operator Lipschitz' or 'operator Hölder' functions, which is very interesting and highly nontrivial for functional calculus for selfadjoint (or, more generally, normal) matrices, becomes trivial 
for extended functional calculus. It seems to be a valid supposition that the main reason for this is that for any compact set $L$ in $\mathbb{C}$ having more than one point and each $k>1$ the closure of $\mathscr{D}_{k}(L)$ is unbounded (and hence noncompact). Taking this into account, it seems to be reasonable to make some restrictions when studying uniform continuity of the extended functional calculus. Let us now state two results in this direction.

5.7. Proposition. For a function $f: \Omega \rightarrow \mathbb{C}$ and $k \geqslant 2$ the following conditions are equivalent:

(i) there is $\varepsilon>0$ such that for any $X_{0} \in \mathscr{D}_{k}(\Omega), f_{\text {op }}: \mathscr{D}_{k}(\Omega) \rightarrow \mathscr{M}_{k}(\mathbb{C})$ is uniformly continuous on the set $\left\{X \in \mathscr{D}_{k}(\Omega):\left\|X-X_{0}\right\|<\varepsilon\right\}$;

(ii) $f_{\text {op }}: \mathscr{D}_{k}(\Omega) \rightarrow \mathscr{M}_{k}(\mathbb{C})$ is uniformly continuous on every bounded subset of $\mathscr{D}_{k}(\Omega)$;

(iii) $f$ extends to a function $F \in \operatorname{DDC}^{k-1}(\bar{\Omega})$.

Moreover, if condition (iii) holds and $F$ is as there, then the function $F_{\text {op }}: \mathscr{M}_{k}^{o}(\bar{\Omega}) \rightarrow$ $\mathscr{M}_{k}(\mathbb{C})$ is uniformly continuous on every bounded subset of $\mathscr{M}_{k}^{o}(\bar{\Omega})$.

Proof. Since the closure (in $\mathscr{M}_{k}(\mathbb{C})$ ) of every bounded subset of $\mathscr{M}_{k}(\mathbb{C})$ is compact, it is easy to check that (ii) follows from (i). Further, if (ii) is fulfilled, it follows from the completeness of $\mathscr{M}_{k}(\mathbb{C})$ that $f_{\text {op }}: \mathscr{D}_{k}(\Omega) \rightarrow \mathscr{M}_{k}(\mathbb{C})$ extends to a continuous function $G: \mathscr{M}_{k}^{o}(\bar{\Omega}) \rightarrow \mathscr{M}_{k}(\mathbb{C})$ (here Lemma 4.2 is applied). Now Proposition 5.1 shows that (iii) is satisfied. Finally, assume $F$ is as in (iii). Then $F_{\text {op }}: \mathscr{M}_{k}^{o}(\bar{\Omega}) \rightarrow$ $\mathscr{M}_{k}(\mathbb{C})$ is continous, by Proposition 4.5 . What is more, $\mathscr{M}_{k}^{o}(\bar{\Omega})$ is closed in $\mathscr{M}_{k}(\mathbb{C})$ (cf. Lemma 4.2) and thus for every bounded set $L \subset \mathscr{M}_{k}^{o}(\bar{\Omega}), F_{\text {op }}$ is uniformly continuous on $L$ (since the closure of $L$ in $\mathscr{M}_{k}^{o}(\bar{\Omega})$ is compact). This proves (i) and completes the proof.

5.8. Proposition. For a function $f: \Omega \rightarrow \mathbb{C}$ and $k \geqslant 2$ the following conditions are equivalent:

(i) for any $z \in \Omega$, there is a relatively open $\left(\right.$ in $\mathscr{D}_{k}(\Omega)$ ) neighbourhood of $z I$ on which $f_{\text {op }}: \mathscr{D}_{k}(\Omega) \rightarrow \mathscr{M}_{k}(\mathbb{C})$ is uniformly continuous;

(ii) every point of $\mathscr{D}_{k}(\Omega)$ has a relatively open $\left(\right.$ in $\left.\mathscr{D}_{k}(\Omega)\right)$ neighbourhood on which the function $f_{\text {op }}: \mathscr{D}_{k}(\Omega) \rightarrow \mathscr{M}_{k}(\mathbb{C})$ is uniformly continuous;

(iii) $f$ extends to a function $\tilde{f} \in \operatorname{DDC}^{k-1}(\tilde{\Omega})$ for some locally compact set $\tilde{\Omega}$ with $\Omega \subset \tilde{\Omega} \subset \bar{\Omega}$.

Moreover, if condition (iii) holds and $\tilde{f}$ is as there, then every point of $\mathscr{M}_{k}^{o}(\tilde{\Omega})$ has a relatively open $\left(\right.$ in $\left.\mathscr{M}_{k}^{o}(\tilde{\Omega})\right)$ neighbourhood on which the function $\tilde{f}_{\text {op }}: \mathscr{M}_{k}^{o}(\tilde{\Omega}) \rightarrow$ $\mathscr{M}_{k}(\mathbb{C})$ is uniformly continuous.

Proof. First assume (iii) holds and let $\tilde{f}$ be as there. Then, according to Proposition $4.5, \tilde{f}_{\text {op }}: \mathscr{M}_{k}^{o}(\tilde{\Omega}) \rightarrow \mathscr{M}_{k}(\mathbb{C})$ is continuous. Since $\tilde{\Omega}$ is locally compact, there is an open (in $\mathbb{C}$ ) set $U$ such that $\tilde{\Omega}=U \cap \bar{\Omega}$. Observe that then $\mathscr{M}_{k}^{o}(\tilde{\Omega})=$ $\mathscr{M}_{k}(U) \cap \mathscr{M}_{k}^{o}(\bar{\Omega})$. But $\mathscr{M}_{k}(U)$ is open in $\mathscr{M}_{k}(\mathbb{C})$ (e.g. by Proposition 4.1), while $\mathscr{M}_{k}^{o}(\bar{\Omega})$ is closed (Lemma 4.2$)$. So, $\mathscr{M}_{k}^{o}(\tilde{\Omega})$, being the intersection of an open and a closed set in the locally compact space $\mathscr{M}_{k}(\mathbb{C})$, is locally compact as well. Consequently, $\tilde{f}_{\text {op }}$ is locally uniformly continuous, which proves (ii) and the additional claim of the proposition. Of course, (i) obviously follows from (ii).

Now assume (i) is fulfilled. For each $z \in \Omega$ let $\varepsilon_{z}>0$ be such that $f_{\text {op }}$ is uniformly continuous on $D_{z}:=B_{z} \cap \mathscr{D}_{k}(\Omega)$ where $B_{z}:=\left\{X \in \mathscr{M}_{k}(\mathbb{C}):\|X-z I\|<\varepsilon_{z}\right\}$. Let $G_{z}: B_{z} \cap \mathscr{M}_{k}^{o}(\bar{\Omega}) \rightarrow \mathscr{M}_{k}(\mathbb{C})$ denote the (unique) continuous extension of $\left.f_{\text {op }}\right|_{D_{z}}$. It then follows that the union of all $G_{z}$ 's is a well defined continuous function on $B \cap \mathscr{M}_{k}^{o}(\bar{\Omega})$ where $B=\bigcup_{z \in \Omega} B_{z}$. Further, let $U=\bigcup_{z \in \Omega} B\left(z, \varepsilon_{z}\right)(\subset \mathbb{C})($ see $(3-2))$ and $\tilde{\Omega}=U \cap \bar{\Omega}$. Notice that $\tilde{\Omega}$ is locally compact and $\Omega \subset \tilde{\Omega}$. It is easily seen that 
$\lambda I \in B$ for any $\lambda \in \tilde{\Omega}$. Moreover, since $G(\lambda I)=\lim _{z \Omega_{\lambda}} f[z I]$ (by the continuity of $G$ ), we see that $G(\lambda I)=w I$ for some $w \in \mathbb{C}$. We define a function $\tilde{f}: \tilde{\Omega} \rightarrow \mathbb{C}$ by the rule: $G(\lambda I)=\tilde{f}(\lambda) I(\lambda \in \tilde{\Omega})$. It is easily seen that $\tilde{f}$ extends $f$. Now we shall show that $\tilde{f} \in \operatorname{DDC}^{k-1}(\tilde{\Omega})$, which will finish the proof.

It is clear that $\tilde{f}$ is continuous. We shall apply this fact in the sequel. Let $\lambda \in \tilde{\Omega}$ and matrices $X_{1}, X_{2}, \ldots \in \mathscr{D}_{k}(\tilde{\Omega})$ converge to $\lambda I$. Let $z \in \Omega$ be such that $\lambda \in B\left(z, \varepsilon_{z}\right)$. Then $\lambda I \in B_{z}$ and hence also $X_{n} \in B_{z}$ for almost all $n$. We may assume $X_{n} \in B_{z}$ for all $n$. It then follows from the continuity of $\tilde{f}$ and the density of $\Omega$ in $\tilde{\Omega}$ that for any $n$ one may find a matrix $X_{n}^{\prime} \in B_{z} \cap \mathscr{D}_{k}(\Omega)$ such that $\left\|X_{n}^{\prime}-X_{n}\right\| \leqslant \frac{1}{n}$ and

$$
\left\|\tilde{f}\left[X_{n}^{\prime}\right]-\tilde{f}\left[X_{n}\right]\right\| \leqslant \frac{1}{n}
$$

(use the diagonalizability argument; cf. the proof of Proposition 5.1). But then $\lim _{n \rightarrow \infty}\left\|X_{n}^{\prime}-\lambda I\right\|=0$ and hence $\tilde{f}\left[X_{n}^{\prime}\right]=f\left[X_{n}^{\prime}\right]=G\left(X_{n}^{\prime}\right) \rightarrow G(\lambda I)=\tilde{f}[\lambda I]$ as $n \rightarrow \infty$, which, combined with (5-8), yields $\lim _{n \rightarrow \infty} \tilde{f}\left[X_{n}\right]=\tilde{f}[\lambda I]$. We now infer from Theorem 4.3 that $\tilde{f} \in \operatorname{DDB}^{k-1}(\tilde{\Omega})$ and $\tilde{f}_{\text {op }}: \mathscr{M}_{k}^{o}(\tilde{\Omega}) \backslash \mathscr{Z}_{k} \rightarrow \mathscr{M}_{k}(\mathbb{C})$ is continuous. In particular,

$$
\tilde{f}[X]=G(X) \quad \text { for any } X \in B \cap \mathscr{M}_{k}^{o}(\tilde{\Omega}) \backslash \mathscr{Z}_{k} .
$$

To ensure that $\tilde{f}$ is of class $\operatorname{DDC}^{k-1}$, it is enough to check that $\tilde{f}_{\text {op }}: \mathscr{D}_{k}(\tilde{\Omega}) \rightarrow \mathscr{M}_{k}(\mathbb{C})$ extends to a continuous function of $\mathscr{M}_{k}^{o}(\tilde{\Omega})$ into $\mathscr{M}_{k}(\mathbb{C})$ (according to Proposition 4.5). Equivalently, we only need to show that if $X_{1}, X_{2}, \ldots$ are arbitrary matrices belonging to $\mathscr{D}_{k}(\tilde{\Omega})$ which converge to some $X \in \mathscr{M}_{k}^{o}(\tilde{\Omega})$, then the sequence $\tilde{f}\left[X_{1}\right], \tilde{f}\left[X_{2}\right], \ldots$ has a limit in $\mathscr{M}_{k}(\mathbb{C})$ (such a criterion for extendability to a continuous function is a general topological fact in metric spaces; see also the last part of the proof of implication '(iv) $\Longrightarrow$ (iii)' in Theorem 4.3). To this end, assume $X_{1}, X_{2}, \ldots \in \mathscr{D}_{k}(\tilde{\Omega})$ converge to $X \in \mathscr{M}_{k}^{o}(\tilde{\Omega})$. If $X \notin \mathscr{Z}_{k}$, then $\lim _{n \rightarrow \infty} \tilde{f}\left[X_{n}\right]=\tilde{f}[X]$, thanks to the previous part of the proof. So, we may assume $X \in \mathscr{Z}_{k}$. Denote by $\lambda \in \tilde{\Omega}$ the unique element of $\operatorname{sp}(X)$. Let $z \in \Omega$ be such that $\lambda \in B\left(z, \varepsilon_{z}\right)$. Then also $\lambda I \in B_{z}$. Denote by $A$ the $k \times k$ matrix $\left[a_{p, q}\right]$ such that $a_{j+1, j}=1(j=1, \ldots, k-1)$ and $a_{p, q}=0$ otherwise. Since $B_{z}$ is open in $\mathscr{M}_{k}(\mathbb{C})$, we see there is $\delta>0$ such that $X^{\prime}:=\lambda I+\delta A \in B_{z}$. Notice that there is an invertible matrix $P \in \mathscr{M}_{k}(\mathbb{C})$ such that $P X P^{-1}=X^{\prime}$ (because $X \in \mathscr{Z}_{k}$ and $\lambda \in \operatorname{sp}(X)$ ). Then the matrices $X_{n}^{\prime}:=P X_{n} P^{-1}$ converge to $X^{\prime}$ and, consequently, belong to $B_{z}$ for almost all $n$. So, $X_{n}^{\prime} \in B \cap \mathscr{M}_{k}^{o}(\tilde{\Omega}) \backslash \mathscr{Z}_{k}, X^{\prime} \in B \cap \mathscr{M}_{k}^{o}(\tilde{\Omega})$ and therefore, by (5-9) and the continuity of $G, \lim _{n \rightarrow \infty} \tilde{f}\left[X_{n}^{\prime}\right]=G\left(X^{\prime}\right)$. Finally, we conclude that $\lim _{n \rightarrow \infty} \tilde{f}\left[X_{n}\right]=\lim _{n \rightarrow \infty} P^{-1} \tilde{f}\left[X_{n}^{\prime}\right] P=P^{-1} G\left(X^{\prime}\right) P$ and we are done.

Now we turn to the second approach to uniform continuity - namely, when the 'quality' of continuity (of the extended functional calculus) is, in a sense, independent of the degree of diagonalizable matrices. The main result in this topic is Theorem 1.3, which we now want to prove.

Proof of Theorem 1.3. Of course, (i) obviously follows from (ii). It is also not so difficult, involving holomorphic functional calculus for bounded Hilbert space operators, that (ii) is implied by (iii). Indeed, denoting by $I$ the identity operator on $\ell_{2}$ (= separable infinite-dimensional complex Hilbert space), it follows from the properties of the holomorphic functional calculus that for any holomorphic function $g: D \rightarrow \mathbb{C}$ (where $D$ is an open neighbourhood of $\lambda$ in $\mathbb{C}$ ) and each $\varepsilon>0$ there is $\delta>0$ such that for every bounded operator $T$ on $\ell_{2}$ with $\|T-\lambda I\| \leqslant \delta$ one has $\operatorname{sp}(T) \subset D$ and $\|f[T]-f[\lambda I]\| \leqslant \varepsilon$ (see also the proof of Proposition 6.4 below). We leave it as an exercise that the assertion of (ii) now easily follows. 
Now assume (i) is fulfilled. We infer from Theorem 4.3 and Proposition 3.2 that $f \in \operatorname{DDC}^{\infty}(\Omega)$. We want to show (iii). To this end, fix $z \in \Omega^{\prime}$ and take $\varepsilon_{z}>0$ and $M_{z}>0$ such that $\|f[X]\| \leqslant M_{z}$ whenever $X \in \mathscr{D}_{n}(\Omega)$ is such that $\left\|X-z I_{n}\right\| \leqslant 2 \varepsilon_{z}$ (where $n$ is arbitrary) - see (i). We claim that for each $n \geqslant 1$,

$$
\begin{aligned}
\left|\Delta\left(\lambda_{1}, \ldots, \lambda_{n}\right) f\right| \leqslant \frac{M_{z}}{\varepsilon_{z}^{n-1}} \quad & \text { if }\left(\lambda_{1}, \ldots, \lambda_{n}\right) \in \Omega^{(n)} \text { and } \\
& \left|\lambda_{j}-z\right| \leqslant \varepsilon_{z}(j=1, \ldots, n) .
\end{aligned}
$$

Observe that the above inequality is immediate for $n=1$. To prove (5-10) for $n>1$, denote by $A$ the $n \times n$ matrix $\left[a_{p, q}\right]$ with $a_{j+1, j}=1(j=1, \ldots, n-1)$ and $a_{p, q}=0$ otherwise, and note that if $\lambda_{1}, \ldots, \lambda_{n}$ are as in (5-10), then $X:=\varepsilon_{z} A+$ $\operatorname{Diag}\left(\lambda_{1}, \ldots, \lambda_{n}\right)$ belongs to $\mathscr{D}_{n}(\Omega),\left\|X-z I_{n}\right\| \leqslant 2 \varepsilon_{z}$ and consequently $\|f[X]\| \leqslant$ $M_{z}$. So, the bottom left corner of $X$, say $b$, satisfies the inequality $|b| \leqslant M_{z}$. But $b=\Delta\left(\lambda_{1}, \ldots, \lambda_{n}\right) f \cdot \varepsilon_{z}^{n-1}$, which yields $(5-10)$.

Since $f \in \operatorname{DDC}^{\infty}(\Omega)$, there are continuous functions $F_{n}: \Omega^{[n]} \rightarrow \mathbb{C}(n=1,2, \ldots)$ such that $F_{n}\left(z_{1}, \ldots, z_{n}\right)=\Delta\left(z_{1}, \ldots, z_{n}\right) f$ for any $\left(z_{1}, \ldots, z_{n}\right) \in \Omega^{(n)}$ (see Proposition 3.3). Further, it follows from Proposition 3.5 and its proof that for any $w \in \Omega$, $z \in \Omega^{\prime}$ and $n \geqslant 1$ :

$$
f(w)=\sum_{k=0}^{n} \frac{f^{(k)}(z)}{k !}(w-z)^{k}+(w-z)^{n}\left[F_{n+1}(z, \ldots, z, w)-F_{n+1}(z, \ldots, z, z)\right] .
$$

The continuity of $F_{n}$ and the density of $\left[B\left(z, \varepsilon_{z}\right)\right]^{n} \cap \Omega^{(n)}$ in $B\left[\left(z, \varepsilon_{z}\right)\right]^{n} \cap \Omega^{[n]}$ combined with (5-10) yield that $\left|F_{n}\left(\lambda_{1}, \ldots, \lambda_{n}\right)\right| \leqslant M_{z} / \varepsilon_{z}^{n-1}$ for any $\left(\lambda_{1}, \ldots, \lambda_{n}\right) \in$ $\left[B\left(z, \varepsilon_{z}\right)\right]^{n} \cap \Omega^{[n]}$ and $z \in \Omega^{\prime}$. So, Taylor's expansion of $f$, stated above, may be estimated as follows:

$$
\left|f(w)-\sum_{k=0}^{n} \frac{f^{(k)}(z)}{k !}(w-z)^{k}\right| \leqslant 2 M_{z}\left(\frac{|w-z|}{\varepsilon_{z}}\right)^{n}
$$

whenever $z \in \Omega^{\prime}, w \in \Omega$ and $|z-w|<\varepsilon_{z}$. Consequently,

$$
f(w)=\sum_{n=0}^{\infty} \frac{f^{(n)}(z)}{n !}(w-z)^{n} \quad\left(z \in \Omega^{\prime}, w \in \Omega,|z-w|<\varepsilon_{z}\right) .
$$

For any $z \in \Omega^{\prime}$ fix $w_{z} \in \Omega$ such that $\delta_{z}:=\left|z-w_{z}\right| \in\left(0, \frac{1}{2} \varepsilon_{z}\right)$. Since the series appearing in (5-11) converges for $w=w_{z}$, we infer that the assignment $w \mapsto$ $\sum_{n=0}^{\infty} \frac{f^{(n)}(z)}{n !}(w-z)^{n}$ well defines a holomorphic function $f_{z}: B\left(z, \delta_{z}\right) \rightarrow \mathbb{C}$ (which extends the restriction of $f$ to $\left.B\left(z, \delta_{z}\right) \cap \Omega\right)$. Put $U=\bigcup_{z \in \Omega^{\prime}} B\left(z, \delta_{z}\right)$. We claim that all the functions $f_{z}\left(z \in \Omega^{\prime}\right)$ agree. Indeed, if the domains of $f_{z_{1}}$ and $f_{z_{2}}$ (for some $z_{1}, z_{2} \in \Omega^{\prime}$ ), that is - the balls $B\left(z_{1}, \delta_{z_{1}}\right)$ and $B\left(z_{2}, \delta_{z_{2}}\right)$, meet, then $\left|z_{1}-z_{2}\right|<\delta_{z_{1}}+\delta_{z_{2}}$. Without loss of generality, we may assume $\delta_{z_{2}} \leqslant \delta_{z_{1}}$. But then $\left|z_{1}-z_{2}\right|<2 \delta_{z_{1}}<\varepsilon_{z_{1}}$ and consequently $z_{2} \in B\left(z_{1}, \varepsilon_{z_{1}}\right) \cap \Omega^{\prime} \cap B\left(z_{2}, \varepsilon_{z_{2}}\right)$. So, the set $A:=B\left(z_{1}, \varepsilon_{z_{1}}\right) \cap B\left(z_{2}, \varepsilon_{z_{2}}\right) \cap \Omega$ has a non-isolated point (namely, $z_{2}$ ) and $\left.f_{z_{1}}\right|_{A}=\left.f\right|_{A}=\left.f_{z_{2}}\right|_{A}$, which implies that $f_{z_{1}}$ and $f_{z_{2}}$ coincide on the whole intersection of their domains (by the identity principle).

The property established above enables us to define a holomorphic function $F: U \rightarrow \mathbb{C}$ by the rule: $F(w)=f_{z}(w)$ for $z \in \Omega^{\prime}$ and $w \in B\left(z, \delta_{z}\right)$. Notice that $F$ extends $\left.f\right|_{\Omega \cap U}$. Hence, if $\Omega \subset U$, the proof is complete. Now assume $\Omega \not \subset U$ and write $U \backslash \Omega=\left\{\lambda_{n}: 1 \leqslant n<N\right\}$ where $N \in\{1,2, \ldots, \infty\}$ and $\lambda_{n}$ 's are different (recall that $U \backslash \Omega$ is finite or countable since $\Omega^{\prime} \subset U$ ). Since $\lambda_{n} \notin \Omega^{\prime}$, there is a real constant $\rho_{n} \in\left(0, \frac{1}{n}\right)$ such that $\Omega \cap B\left(\lambda_{n}, \rho_{n}\right)=\left\{\lambda_{n}\right\}(n<N)$. Notice that the sets

$$
U_{0}:=U \backslash \overline{\bigcup_{n<N} B\left(\lambda_{n}, \frac{1}{2} \rho_{n}\right)}, B\left(\lambda_{1}, \frac{1}{2} \rho_{1}\right), B\left(\lambda_{2}, \frac{1}{2} \rho_{2}\right), \ldots
$$


are open and pairwise disjoint and hence we may properly define a holomorphic function $g: D \rightarrow \mathbb{C}$ on their union $D$ by the rule: $g(w)=F(w)$ for $w \in U_{0}$ and $g(w)=f\left(\lambda_{n}\right)$ for $w \in B\left(\lambda_{n}, \frac{1}{2} \rho_{n}\right)(n<N)$. It is clear that $g$ extends $\left.f\right|_{\Omega \cap D}$. So, to finish the proof, it suffices to check that $\Omega \subset D$. Suppose, on the contrary, that there is $z \in \Omega$ which does not belong to $D$. Then $z \neq \lambda_{n}(n<N)$ and thus $z \in U$. We conclude from the fact that $z \in U \backslash D$ that $z$ belongs to the closure of $\bigcup_{n<N} B\left(\lambda_{n}, \frac{1}{2} \rho_{n}\right)$. So, there are sequences $\left(n_{k}\right)_{k=1}^{\infty}$ and $\left(z_{k}\right)_{k=1}^{\infty}$ of natural and complex numbers (respectively) such that $z_{k} \in B\left(\lambda_{n_{k}}, \frac{1}{2} \rho_{n_{k}}\right)$ and $\lim _{k \rightarrow \infty} z_{k}=$ $z$. Passing to a subsequence, we may assume that either $n_{k}=m$ for all $k$ or $\lim _{k \rightarrow \infty} n_{k}=\infty$. In the first case we obtain that $\left|z-\lambda_{m}\right| \leqslant \frac{1}{2} \rho_{m}$ and thus $z \in B\left(\lambda_{m}, \rho_{m}\right) \cap \Omega$, which is impossible (since $\left.z \neq \lambda_{m}\right)$. In the second case we infer that $\lim _{k \rightarrow \infty} \lambda_{n_{k}}=z(\in U)$ because $\left|z_{n_{k}}-\lambda_{n_{k}}\right|<\rho_{n_{k}}<\frac{1}{n_{k}}$. So, there is $l$ such that $\lambda_{n_{l}} \in U$, which is also impossible, and we are done.

\section{Functional Calculus in infinite dimension}

Now we would like to introduce and study extended functional calculus for bounded operators in infinite-dimensional (complex) Hilbert spaces. To this end, let us fix the notation. Whenever $\mathcal{H}$ is a Hilbert space, by $\mathcal{B}(\mathcal{H})$ we denote the unital $\left(C^{*}\right.$-)algebra of all bounded linear operators acting on $\mathcal{H}$. The spectrum of $T \in \mathcal{B}(\mathcal{H})$ is the set $\operatorname{sp}(T)$ of all scalars $\lambda \in \mathbb{C}$ such that the operator $T-\lambda I$ is noninvertible in $\mathcal{B}(\mathcal{H})$ (here $I$ denotes the identity operator on $\mathcal{H}$ ). Two bounded operators $S$ and $T$ are said to be similar if there is an invertible operator $G \in \mathcal{B}(\mathcal{H})$ such that $T=G S G^{-1}$.

6.1. Definition. Let $\mathcal{H}$ be a Hilbert space with a fixed orthonormal basis $\mathscr{B}=$ $\left\{e_{j}\right\}_{j \in J}$. An operator $T \in \mathcal{B}(\mathcal{H})$ is said to be diagonal (with respect to $\mathscr{B}$ ) iff $T e_{j} \in \mathbb{C} \cdot e_{j}$ for any $j \in J$. $T$ is called diagonalizable iff it is similar to diagonal. Finally, $T$ is scalar (in the sense of Dunford and Schwartz [6]) if it is similar to a normal operator.

The sets of all (bounded) diagonalizable and scalar operators on $\mathcal{H}$ are denoted by, respectively, $\mathscr{D}_{\mathcal{H}}$ and $\mathscr{S}_{\mathcal{H}}$.

The original definition of a scalar operator due to Dunford and Schwartz [6] differs from ours. However, they are equivalent (which was also established by Dunford and Schwartz, see [6, Theorem XV.6.4]). It is easily seen that the definition of a diagonal operator does depend on the choice of an orthonormal basis, while the notion of a diagonalizable operator does not. That is, if $T$ is similar to a diagonal operator with respect to an orthonormal basis $\mathscr{B}$, then $T$ is similar to a diagonal operator with respect to any other orthonormal basis as well. Finally, since diagonal operators are normal, we see that diagonalizable operators are scalar. So, $\mathscr{D}_{\mathcal{H}} \subset \mathscr{S}_{\mathcal{H}}$. Our aim is to define extended functional calculus for scalar operators. To this end, we recall that for every normal operator $N \in \mathcal{B}(\mathcal{H})$ there exists a unique spectral measure $E: \mathfrak{B}(\operatorname{sp}(N)) \rightarrow \mathcal{B}(\mathcal{H})$ (where $\mathfrak{B}(A)$ is the $\sigma$-algebra of all Borel subsets of a compact set $A \subset \mathbb{C}$ ) such that $N=\int_{\operatorname{sp}(N)} z E(\mathrm{~d} z)$ (this fact is known as the spectral theorem). Then, for every bounded Borel function $f: \operatorname{sp}(N) \rightarrow \mathbb{C}$ one defines $f[N]$ as the integral $\int_{\operatorname{sp}(N)} f(z) E(\mathrm{~d} z)$. With the aid of the Fuglede-Putnam theorem [7, 12] (see also [5, Theorem IX.6.7] for a simpler proof) one easily proves the following result on intertwining between normal operators.

6.2. Lemma. Let $N, M \in \mathcal{B}(\mathcal{H})$ be normal and $P, G \in \mathcal{B}(\mathcal{H})$ be invertible operators. If $P N P^{-1}=G M G^{-1}$, then $\operatorname{sp}(N)=\operatorname{sp}(M)$ and

$$
\operatorname{Pf}[N] P^{-1}=G f[M] G^{-1}
$$

for any bounded Borel function $f: \operatorname{sp}(N) \rightarrow \mathbb{C}$. 
Lemma 6.2 enables us to define extended functional calculus for scalar operators. Let us introduce

6.3. Definition. For any set $\Omega \subset \mathbb{C}$ and a Hilbert space $\mathcal{H}$ denote by $\mathscr{D}_{\mathcal{H}}(\Omega)$ and $\mathscr{S}_{\mathcal{H}}(\Omega)$ the sets of all diagonalizable and, respectively, scalar operators on $\mathcal{H}$ whose spectra are contained in $\Omega$.

Let $f: \Omega \rightarrow \mathbb{C}$ be a Borel function which is bounded on compact subsets of $\Omega$. For any operator $T \in \mathscr{S}_{\mathcal{H}}(\Omega)$ we define $f[T]$ as follows: take an invertible operator $G \in \mathcal{B}(\mathcal{H})$ such that $G T G^{-1}$ is normal and put

$$
f[T]=G^{-1} f\left[G T G^{-1}\right] G .
$$

Lemma 6.2 asserts that $f[T]$ is well defined, i.e. it is independent of the choice of $G$ for which $G T G^{-1}$ is normal.

When $g(z)=\bar{z}$, the transform $T \mapsto g[T]$ (where $T$ runs over scalar operators) was studied by us earlier [11], where we used other notation. For any scalar operator $T, g[T]$ was denoted by $T^{(*)}$ and called the quasi-adjoint of $T$. (The quasi-adjoint was involved there to characterize operator algebras similar to commutative $C^{*}$ algebras.)

Our last aim of the paper is to characterize those functions $f$ for which the transform $f_{\text {op }}: \mathscr{D}_{\mathcal{H}}(\Omega) \ni T \mapsto f[T] \in \mathcal{B}(\mathcal{H})$ is continuous on some (or any) infinitedimensional Hilbert space $\mathcal{H}$. This is included in the next result, where we denote by $\ell_{2}$ the classical separable Hilbert space. As usual, $I$ stands for the identity operator on a suitable Hilbert space.

6.4. Proposition. For a continuous function $f: \Omega \rightarrow \mathbb{C}$ the following conditions are equivalent:

(i) for each $\lambda \in \Omega^{\prime}$ there exist positive real constants $\varepsilon=\varepsilon(\lambda)$ and $M=M(\lambda)$ such that $\|f[K+\lambda I]\| \leqslant M$ whenever $K \in \mathcal{B}\left(\ell_{2}\right)$ is a finite-dimensional diagonalizable operator such that $\|K\| \leqslant \varepsilon$ and $\operatorname{sp}(K+\lambda I) \subset \Omega$;

(ii) $f_{\text {op }}: \mathscr{D}_{\ell_{2}}(\Omega) \rightarrow \mathcal{B}\left(\ell_{2}\right)$ is continuous;

(iii) for an arbitrary Hilbert space $\mathcal{H}$, the function $f_{\text {op }}: \mathscr{S}_{\mathcal{H}}(\Omega) \rightarrow \mathcal{B}(\mathcal{H})$ extends to a continuous function of the set $\{T \in \mathcal{B}(\mathcal{H}): \operatorname{sp}(T) \subset D\}$ for some open (in $\mathbb{C})$ set $D$;

(iv) $f$ extends to a holomorphic function of an open set $D \supset \Omega$.

Proof. First assume $f$ extends to a holomorphic function $F: D \rightarrow \mathbb{C}$. It follows from the Riesz functional calculus that $F$ induces a holomorphic function $F_{\text {op }}: U \rightarrow \mathcal{B}(\mathcal{H})$ on the open (in $\mathcal{B}(\mathcal{H})$ ) set $U:=\{T \in \mathcal{B}(\mathcal{H}): \operatorname{sp}(T) \subset D\}$ (consult e.g. [5, VII. $\S 4]$ or $[3$, I. $\S 7])$. It is easy to check that $F_{\text {op }}$ extends $f_{\text {op }}: \mathscr{S}_{\mathcal{H}}(\Omega) \rightarrow \mathcal{B}(\mathcal{H})$. This shows that (iii) follows from (iv). Since (i) obviously follows from (ii), and (ii) from (iii), we only need to prove that (iv) is implied by (i). Taking into account Theorem 1.3, it suffices to verify that condition (i) of that result is satisfied. But that condition easily follows from the condition (i) of the proposition. The details are left to the reader.

The above result has a remarkable consequence: the Riesz functional calculus (that is, the holomorphic functional calculus) is as wide as possible when we require its continuity.

\section{REFERENCES}

[1] A.B. Aleksandrov, V.V. Peller, D.S. Potapov and F.A. Sukochev, Functions of normal operators under perturbations, Adv. Math. 226 (2011), 5216-5251.

[2] R. Bhatia, Matrix Analysis, Springer, New York, 1997.

[3] F.F. Bonsall and N.J. Duncan, Complete Normed Algebras, Springer-Verlag, Berlin, 1973.

[4] C. de Boor, Divided differences, Surv. Approx. Theory 1 (2005), 46-69. 
[5] J.B. Conway, A Course in Functional Analysis (Graduate Texts in Mathematics, vol. 96), Springer, New York, 1990.

[6] N. Dunford and J.T. Schwartz, Linear Operators, part III, Wiley-Interscience, New York, 1971.

[7] B. Fuglede, A commutativity theorem for normal operators, Proc. Natl. Acad. Sci. USA 36 (1950), 35-40.

[8] T. Kato, Continuity of the map $S \mapsto|S|$ for linear operators, Proc. Japan Acad. 49 (1973), $157-160$.

[9] K. Löwner, Über monotone Matrixfunctionen, Math. Z. 38 (1934), 177-216.

[10] G. Opitz, Steigungsmatrizen, Z. Angew. Math. Mech. 44 (1964), T52-T54.

[11] P. Niemiec, Separate and joint similarity to families of normal operators, Studia Math. 149 (2002), 39-62.

[12] C.R. Putnam, On normal operators in Hilbert space, Amer. J. Math. 73 (1951), 357-362.

[13] D. Serre, Matrices: Theory and Applications (Graudate Texts in Mathematics 216), Springer-Verlag, New York, 2002.

[14] H. Whitney, Derivatives, difference quotients and Taylor's formula, Bull. Amer. Math. Soc. 40 (1934), 89-94.

[15] H. Whitney, Differentiable functions defined in closed sets. I, Trans. Amer. Math. Soc. 36 (1934), 369-387.

Piotr Niemiec, Instytut Matematyki, Wydzią Matematyki i Informatyki, UniWersyTET Jagielloński, ul. ŁoJASIEWicza 6, 30-348 Kraków, Poland

E-mail address: piotr.niemiec@uj.edu.pl 